\title{
ANALYSIS OF A BACKWARD EULER-TYPE SCHEME FOR MAXWELL'S EQUATIONS IN A HAVRILIAK-NEGAMI DISPERSIVE MEDIUM
}

\author{
Yubo YAnG ${ }^{1}$, Li-Lian WAnG ${ }^{2, *}$ AND FAnHAI ZENG ${ }^{3}$
}

\begin{abstract}
For the Maxwell's equations in a Havriliak-Negami (H-N) dispersive medium, the associated energy dissipation law has not been settled at both continuous level and discrete level. In this paper, we rigorously show that the energy of the H-N model can be bounded by the initial energy and the model is well-posed. We analyse a backward Euler-type semi-discrete scheme, and prove that the modified discrete energy decays monotonically in time. Such a strong stability ensures that the scheme is unconditionally stable. We also introduce a fast temporal convolution algorithm to alleviate the burden of the history dependence in the polarisation relation involving the singular kernel with the Mittag-Leffler function with three parameters. We provide ample numerical results to demonstrate the efficiency and accuracy of a full-discrete scheme via a spectra-Galerkin method in two dimensions. Finally, we consider an interesting application in the recovery of complex relative permittivity and some related physical quantities.
\end{abstract}

Mathematics Subject Classification. 65N35, 65E05, 65N12, 41A10, 41A25, 41A30, 41A58.

Received July 15, 2020. Accepted January 21, 2021.

\section{INTRODUCTION}

In electromagnetism, the most general model for a dispersive dielectric material, i.e. a material with frequencydependent permittivity, is the Havriliak-Negami (H-N) dielectric model (see, e.g. [19,20,22,37]). In this model, the complex relative permittivity is expressed as

$$
\epsilon_{r}(\omega)=\epsilon_{\infty}+\frac{\epsilon_{s}-\epsilon_{\infty}}{\left(1+\left(i \omega \tau_{0}\right)^{\alpha}\right)^{\beta}}
$$

where $0<\alpha, \beta \leq 1, \epsilon_{\infty}, \epsilon_{s}$ and $\tau_{0}$ are the infinite-frequency permittivity, the static permittivity and the relaxation time respectively, and $\epsilon_{s}, \epsilon_{\infty}$ satisfy $\epsilon_{s}>\epsilon_{\infty} \geq 1$. Furthermore, $i=\sqrt{-1}$ denotes the imaginary unit, and $\omega$ is the angular frequency. All the anomalously dispersive dielectric models are its subclasses. When $\alpha=\beta=1$, the H-N model reduces to the Debye model [10], while the H-N model reduces to Davidson-Cole

Keywords and phrases. Maxwell's equations, Havriliak-Negami dispersive medium, strong stability, unconditionally stable scheme, fast temporal convolution algorithm.

1 Nanhu College, Jiaxing University, 314001 Jiaxing, Zhejiang, P.R. China.

2 Division of Mathematical Sciences, School of Physical and Mathematical Sciences, Nanyang Technological University, 637371, Singapore.

3 School of Mathematics, Shandong University, 250100 Jinan, Shandong, P.R. China.

* Corresponding author: lilian@ntu.edu.sg 
(D-C) model [9] when $\alpha=1$, and to Cole-Cole (C-C) model when $\beta=1$ [8]. Such models arise from diverse fields, which typically include biological tissues [4,27,34], soils [41], amorphous polymers near the glass-liquid transition [13], glassy materials [1] among others.

In general, there are two main strategies to simulate the electromagnetic wave propagations in dispersive media based on different treatments of the relation between the electric flux and electric field intensity, governed by the polarisation equation. The first is to introduce certain auxiliary function and related auxiliary differential equation (ADE) to deal with the polarisation. The second is to formulate the polarisation as a time convolution integral equation of the electric field. For the Debye or Debye-type model, such as Drude or Lorentz model, its time-domain expression of its relative complex permittivity can be easily formulated, because its relative complex permittivity is a function of integer powers of $i \omega$. Therefore, both approaches can be applied. In particular, the ADE involves derivatives of integer order that can be discretised by the finite difference methods $[12,45]$ as usual. However, the relative complex permittivity of the H-N, D-C or C-C model involves a function of noninteger powers of $i \omega$, so its representation in the time domain is much more complicated. In fact, the polarisation relations are oftentimes integro-differential equations with global fractional operators $[5,21,26,33,40]$, which pose significant difficulties and are much more expensive to solve.

In regards to the C-C model, fractional ADE-based time-domain methods were proposed in e.g. [26,38,43, 46], where the polarisation equation involves the fractional-in-time Riemann-Liouville derivative ( $c f$. [33]). As such, much recent development in numerical fractional differential equations casts light on time discretisation of this model. However, there has been very limited works on numerical solutions of the D-C and H-N models, largely due to that the polarisation relation cannot be expressed in terms of ADE with usual fractional differential operators. Nevertheless, some interesting attempts include the approximation of the D-C or H-N model by the Debye model in the frequency domain $[6,23,39,40]$; or by the C-C model in the frequency domain $[2,3,30,42]$. We remark that most works related to H-N, C-C or D-C model above are implemented by the finite difference time-domain (FDTD) method ( $c f .[45])$, and the stability and convergence analysis is yet unavailable. On the other hand, Li et al. [26], and Huang and Wang [21] developed a finite element time-domain (FETD) method (based on a fractional differential form of the polarisation equation) and a spectral time-domain method (based on an integro-differential formulation) for the C-C model, respectively. Stability and convergence analysis were also provided in these two works.

In this paper, we propose and analyse a time-domain numerical method for solving the H-N model with the polarisation relation formulated by an integral equation involving a singular kernel function in terms of the Mittag-Leffler (ML) function with three parameters. We highlight our main contributions as follows.

- With the aid of some useful properties of the ML function, we prove that the energy of the H-N model can be controlled by the initial energy, which ensures the well-posedness of the model and plays an important role in developing stable numerical methods.

- We conduct a delicate and rigorous analyse of a semi-discrete scheme which can incorporate various spatial discretisation. More precisely, we propose a first-order backward-Euler-type scheme, and show for the first time that the discrete energy (with a modification of the continuous energy by adding a history part) decays monotonically. This strong stability guarantees that the scheme is unconditionally stable and is essential for the convergence analysis. However, it appears nontrivial to show this if one works with the fractional differential form of the polarisation relation in the context of the C-C model [26].

- A fast temporal convolution algorithm for the H-N model is realised by following some basic ideas in $[28,48]$, which requires $O\left(\log N_{t}\right)$ storage and $O\left(N_{t} \log N_{t}\right)$ operations over $N_{t}$ time steps, when only cost in time direction is considered. Here, $N_{t}$ represents the total number of time steps. Note that the direct implementation of the scheme (3.2) would require $O\left(N_{t}\right)$ storage and $O\left(N_{t}^{2}\right)$ operations, which is computational expensive and forms a bottleneck for long time simulation.

The rest of this paper is organised as follows. In the next section, we introduce the Havriliak-Negami dispersive dielectric model, and conduct the stability analysis. In Section 3, we propose a time discrete scheme for the H-N model, and provide its stability and error analysis. In Section 4, we implement a fast temporal convolution 
algorithm, and illustrate spatial discretisation through a two-dimensional H-N model. Then we supply with ample numerical results to demonstrate the efficiency and accuracy of the proposed scheme. Furthermore, we apply the solver to an interesting application in the recovery of the complex relative permittivity and some related physical quantities. Finally, concluding remarks are made in Section 5, and some important properties of the Mittag-Leffler function are collected in Appendix A.

At the end of this section, we introduce some notations to be used throughout the paper. Let $C$ (sometimes with a subindex) denote a generic constant independent of the time step size $\Delta t$ and the space parameter $N$. For $r \geq 0$, let $H^{r}(\Omega)\left(\right.$ resp. $\left.\boldsymbol{H}^{r}(\Omega)\right)$ be the usual Sobolev space with $H^{0}(\Omega)=L^{2}(\Omega)\left(\operatorname{resp} . \boldsymbol{H}^{0}(\Omega)=\boldsymbol{L}^{2}(\Omega)\right.$ ) for the scalar (resp. vector-valued) functions on a bounded domain $\Omega$ with Lipschitz boundary. As usual, we denote the inner product and norm of both $L^{2}(\Omega)$ and $\boldsymbol{L}^{2}(\Omega)$ by $(\cdot, \cdot)$ and $\|\cdot\|$, respectively. With a little abuse of notation, we denote the norms of both $L^{\infty}(\Omega)$ and $\boldsymbol{L}^{\infty}(\Omega)$ by $\|\cdot\|_{\infty}$. Given a Hilbert space $\boldsymbol{X}$ with the norm $\|\cdot\|_{\boldsymbol{X}}$, we define the spaces $L^{\infty}(0, T ; \boldsymbol{X})$ and $L^{2}(0, T ; \boldsymbol{X})$ with the norms

$$
\|\boldsymbol{U}\|_{L^{\infty}(0, T ; \boldsymbol{X})}=\operatorname{esssup}_{0 \leq t \leq T}\|\boldsymbol{U}(\cdot, t)\|_{\boldsymbol{X}}, \quad\|\boldsymbol{U}\|_{L^{2}(0, T ; \boldsymbol{X})}=\left(\int_{0}^{T}\|\boldsymbol{U}(\cdot, t)\|_{\boldsymbol{X}}^{2} \mathrm{~d} t\right)^{2} .
$$

We further introduce

$$
H^{k}(0, T ; \boldsymbol{X}) \triangleq\left\{\boldsymbol{v} \in L^{2}(0, T ; \boldsymbol{X}): \partial_{t}^{\ell} \boldsymbol{v} \in L^{2}(0, T ; \boldsymbol{X}), 1 \leq \ell \leq k\right\}
$$

with the norm $\|\cdot\|_{H^{k}(0, T ; \boldsymbol{X})}(c f .[36])$. We also use some common notation (cf. [31])

$$
H(\operatorname{curl} ; \Omega)=\left\{\boldsymbol{v} \in \boldsymbol{L}^{2}(\Omega) ; \nabla \times \boldsymbol{v} \in \boldsymbol{L}^{2}(\Omega)\right\}, \quad H_{0}(\operatorname{curl} ; \Omega)=\{\boldsymbol{v} \in H(\operatorname{curl} ; \Omega) ; \boldsymbol{n} \times \boldsymbol{v}=0 \text { on } \partial \Omega\} .
$$

\section{The HavriliaK-Negami Dispersive DieleCtric MODEL}

In an H-N medium, the time-domain Maxwell's equations take the form (cf. $[19,20])$ :

$$
\epsilon_{0} \epsilon_{\infty} \partial_{t} \boldsymbol{E}=\nabla \times \boldsymbol{H}-\partial_{t} \boldsymbol{P}, \quad \mu_{0} \partial_{t} \boldsymbol{H}=-\nabla \times \boldsymbol{E} \quad \text { in } \Omega \times(0, T],
$$

where $\boldsymbol{P}(\boldsymbol{x}, t)$ is the induced electric polarisation given by

$$
\boldsymbol{P}(\boldsymbol{x}, t)=\int_{0}^{t} \xi_{\alpha, \beta}(t-s) \boldsymbol{E}(\boldsymbol{x}, s) \mathrm{d} s, \quad \forall(\boldsymbol{x}, t) \in \Omega \times(0, T] .
$$

Here $\xi_{\alpha, \beta}$ is the time-domain susceptibility kernel which involves the inverse Laplace transform as follows

$$
\xi_{\alpha, \beta}(t):=\mathscr{L}^{-1}\left[\frac{\epsilon_{0}\left(\epsilon_{s}-\epsilon_{\infty}\right)}{\left(1+\left(s \tau_{0}\right)^{\alpha}\right)^{\beta}}\right](t),
$$

and $\tau_{0}, \epsilon_{s}, \epsilon_{\infty}, \alpha, \beta$ are given in (1.1). As usual, $\boldsymbol{E}$ is the electric field, $\boldsymbol{H}$ is the magnetic field, and $\epsilon_{0}, \mu_{0}$ are the permittivity and permeability of the free space, respectively. The system (2.1) and (2.2) is supplemented with the perfect electrical conductor (PEC) condition

$$
\boldsymbol{n} \times \boldsymbol{E}=\mathbf{0} \quad \text { at } \partial \Omega \times(0, T],
$$

and the initial conditions

$$
\boldsymbol{E}(\boldsymbol{x}, 0)=\boldsymbol{E}_{0}(\boldsymbol{x}), \quad \boldsymbol{H}(\boldsymbol{x}, 0)=\boldsymbol{H}_{0}(\boldsymbol{x}), \quad \boldsymbol{P}(\boldsymbol{x}, 0)=\mathbf{0} \quad \text { in } \Omega,
$$

where the last condition is a direct consequence of the representation (2.2). Here the constitutive relations in an $\mathrm{H}-\mathrm{N}$ medium are

$$
\boldsymbol{D}=\epsilon_{0} \epsilon_{\infty} \boldsymbol{E}+\boldsymbol{P}, \quad \boldsymbol{B}=\mu_{0} \boldsymbol{H}
$$


where $\boldsymbol{D}$ is the electric flux density, and $\boldsymbol{B}$ is the magnetic flux density.

As the values of the parameters $\epsilon_{0}, \mu_{0}$ and $\tau_{0}$ are excessively small (of order $10^{-12}, 10^{-7}$ and $10^{-12}$, respectively), we find it is more desirable to rescale the model for both computational and analysis purposes. Indeed, the introduction of non-dimensional quantities can avoid dealing with excessively small or large numbers in finite-precision arithmetic (cf. [11], P. 294).

Lemma 2.1. Using the substitutions and change of variables

$$
\boldsymbol{E} \rightarrow \sqrt{\epsilon_{0}} \boldsymbol{E}, \quad \boldsymbol{P} \rightarrow \frac{1}{\sqrt{\epsilon_{0}}} \boldsymbol{P}, \quad \boldsymbol{H} \rightarrow \sqrt{\mu_{0}} \boldsymbol{H}, \quad t \rightarrow \frac{t}{\tau_{0}}, \quad \boldsymbol{x} \rightarrow \frac{\boldsymbol{x}}{c_{0} \tau_{0}}, \quad c_{0}:=\frac{1}{\sqrt{\epsilon_{0} \mu_{0}}},
$$

we can convert the system (2.1)-(2.5) into

$$
\left\{\begin{array}{l}
\epsilon_{\infty} \partial_{t} \boldsymbol{E}+\partial_{t} \boldsymbol{P}=\nabla \times \boldsymbol{H}, \quad \partial_{t} \boldsymbol{H}=-\nabla \times \boldsymbol{E} \quad \text { in } \Omega \times(0, T], \\
\boldsymbol{P}(\boldsymbol{x}, t)=\Delta \epsilon \int_{0}^{t} e_{\alpha, \alpha \beta}^{\beta}(t-s ;-1) \boldsymbol{E}(\boldsymbol{x}, s) \mathrm{d} s \quad \text { in } \Omega \times(0, T], \\
\boldsymbol{E}(\boldsymbol{x}, 0)=\boldsymbol{E}_{0}(\boldsymbol{x}), \quad \boldsymbol{H}(\boldsymbol{x}, 0)=\boldsymbol{H}_{0}(\boldsymbol{x}), \quad \boldsymbol{P}(\boldsymbol{x}, 0)=\mathbf{0} \quad \text { in } \Omega, \\
\boldsymbol{n} \times \boldsymbol{E}=\mathbf{0} \quad \text { at } \partial \Omega \times(0, T],
\end{array}\right.
$$

where $\Delta \epsilon:=\epsilon_{s}-\epsilon_{\infty}$, and

$$
e_{\rho, \mu}^{\gamma}(t ; \sigma)=t^{\mu-1} E_{\rho, \mu}^{\gamma}\left(\sigma t^{\rho}\right), \quad E_{\rho, \mu}^{\gamma}(z)=\sum_{k=0}^{\infty} \frac{\Gamma(k+\gamma)}{\Gamma(k) \Gamma(\rho k+\mu)} \frac{z^{k}}{k !},
$$

i.e. the Mittag-Leffler $(M L)$ function with three parameters (also known as the Prabhakar function, see [18, 35] and Appendix A).

Proof. One verifies readily that with (2.6), the rescaled system (2.7) (except for (2.7b)) can be reduced from (2.1), (2.4) and (2.5) directly.

Now, we consider the derivation of (2.7b). According to (A.1) and (2.3), we have

$$
\xi_{\alpha, \beta}(t)=\frac{\epsilon_{0} \Delta \epsilon}{\tau_{0}^{\alpha \beta}} \mathscr{L}^{-1}\left[\left(s^{\alpha}+1 / \tau_{0}^{\alpha}\right)^{-\beta}\right](t)=\frac{\epsilon_{0} \Delta \epsilon}{\tau_{0}^{\alpha \beta}} e_{\alpha, \alpha \beta}^{\beta}\left(t ;-\frac{1}{\tau_{0}^{\alpha}}\right) .
$$

Then (2.2) can be written as

$$
\boldsymbol{P}(\boldsymbol{x}, t)=\frac{\epsilon_{0} \Delta \epsilon}{\tau_{0}^{\alpha \beta}} \int_{0}^{t} e_{\alpha, \alpha \beta}^{\beta}\left(t-s ;-1 / \tau_{0}^{\alpha}\right) \boldsymbol{E}(\boldsymbol{x}, s) \mathrm{d} s .
$$

With the substitution $s \rightarrow \frac{s}{\tau_{0}}$ and $t \rightarrow \frac{t}{\tau_{0}}$, we can obtain (2.7b) from the above.

Formally, the rescaled polarisation relation $(2.7 \mathrm{~b})$ can be reformulated as a fractional "differential" form using the Prabhakar integrals/derivatives $(c f .[16,17])$, which turns out to be important for the stability analysis of the re-scaled model (2.7).

Definition 2.2 (see [16], (B.19)-(B.23) or [17], (5.3)-(5.10)). For a function $f(t) \in L^{1}(0, T)$, the Prabhakar integral of order $\alpha, \beta>0$ and with the parameter $\varrho>0$ can be defined by

$$
\left({ }_{0} \mathcal{J}_{t}^{\alpha}+\varrho\right)^{\beta} f(t)=\int_{0}^{t} e_{\alpha, \alpha \beta}^{\beta}(t-s ;-\varrho) f(s) \mathrm{d} s, \quad t \in(0, T) .
$$


If, in addition, $0<\alpha \beta<1$, the left-inverse of the above integral operator is the special derivative

$$
\left({ }_{0} \mathcal{D}_{t}^{\alpha}+\varrho\right)^{\beta} f(t)=\frac{\mathrm{d}}{\mathrm{d} t} \int_{0}^{t} e_{\alpha, 1-\alpha \beta}^{-\beta}(t-s ;-\varrho) f(s) \mathrm{d} s .
$$

For an absolutely continuous function $f(t)$, the Caputo-type derivative as the counterpart of the above derivative operator can be defined as

$$
{ }^{C}\left({ }_{0} \mathcal{D}_{t}^{\alpha}+\varrho\right)^{\beta} f(t)=\left({ }_{0} \mathcal{D}_{t}^{\alpha}+\varrho\right)^{\beta}\left(f(t)-f\left(0^{+}\right)\right)=\int_{0}^{t} e_{\alpha, 1-\alpha \beta}^{-\beta}(t-s ;-\varrho) f^{\prime}(s) \mathrm{d} s .
$$

In view of $(2.10)$ with $\varrho=1$, we can write $(2.7 \mathrm{~b})$ as

$$
\boldsymbol{P}(\boldsymbol{x}, t)=\Delta \epsilon\left({ }_{0} \mathcal{J}_{t}^{\alpha}+1\right)^{\beta} \boldsymbol{E}(\boldsymbol{x}, t),
$$

Taking the left-inverse operation (2.11) on both sides of (2.13), we obtain immediately from (2.12) that

$$
\left({ }_{0} \mathcal{D}_{t}^{\alpha}+1\right)^{\beta} \boldsymbol{P}(\boldsymbol{x}, t)={ }^{C}\left({ }_{0} \mathcal{D}_{t}^{\alpha}+1\right)^{\beta} \boldsymbol{P}(\boldsymbol{x}, t)=\Delta \epsilon \boldsymbol{E}(\boldsymbol{x}, t) .
$$

It is noteworthy that when $\beta=1$ (i.e. the $\mathrm{C}-\mathrm{C}$ model), the involved fractional derivatives simply become the usual fractional Riemann-Liouville derivative and Caputo derivative operators as in [33]. In fact, fractional ADE-based approaches for the C-C model are based upon such a formulation. However, for the general H-N model, we find the integral formulation (2.13) is more suitable for the implementation, but the formulation (2.14) is useful in the analysis.

Lemma 2.3. If $0<\alpha, \beta \leq 1$ and $\varrho>0$, then the kernel $e_{\alpha, 1-\alpha \beta}^{-\beta}(t ;-\varrho)$ in (2.11) and (2.14) is positive-definite in the sense that

$$
\int_{0}^{T} \phi(t) \int_{0}^{t} e_{\alpha, 1-\alpha \beta}^{-\beta}(t-s ;-\varrho) \phi(s) \mathrm{d} s \mathrm{~d} t \geq 0, \quad \forall \phi \in C[0, T] .
$$

Proof. According to (1.2) of [29], it suffices to show that the kernel function $\mathcal{K}(t):=e_{\alpha, 1-\alpha \beta}^{-\beta}(t ;-\varrho)$ satisfies

$$
\operatorname{Re}\{\mathscr{L}[\mathcal{K}(t)](i \omega)\} \geq 0, \quad \forall \omega>0,
$$

where $\operatorname{Re}\{u\}$ stands for the real part of $u$ and $i$ is the imaginary unit. Using (A.1) with $\gamma=-\beta, \rho=\alpha, \mu=$ $1-\alpha \beta, \sigma=\varrho$ and $s=i \omega$, we find from direct calculation that

$$
\begin{aligned}
\mathscr{L}[\mathcal{K}(t)](i \omega) & =\frac{(i \omega)^{\alpha(-\beta)-(1-\alpha \beta)}}{\left((i \omega)^{\alpha}+\varrho\right)^{-\beta}}=\frac{\left(\varrho+(i \omega)^{\alpha}\right)^{\beta}}{i \omega}=-i \omega^{-1}\left(\varrho+\omega^{\alpha} \cos \frac{\pi \alpha}{2}+i \omega^{\alpha} \cos \frac{\pi \alpha}{2}\right)^{\beta} \\
& =-i \omega^{-1} r^{\beta}(\cos \beta \theta+i \sin \beta \theta)=\omega^{-1} r^{\beta}(\sin \beta \theta-i \cos \beta \theta)
\end{aligned}
$$

where

$$
r=\sqrt{\varrho^{2}+2 \omega^{\alpha} \cos \frac{\pi \alpha}{2}+\omega^{2 \alpha}}, \quad \tan \theta=\frac{\omega^{\alpha} \sin \frac{\pi \alpha}{2}}{\varrho+\omega^{\alpha} \cos \frac{\pi \alpha}{2}} .
$$

As the parameters $0<\alpha, \beta \leq 1$ and $\varrho>0$, it is evident that $\theta \in(0, \pi / 2)$. Therefore,

$$
\operatorname{Re}\{\mathscr{L}[\mathcal{K}(t)](i \omega)\}=\omega^{-1} r^{\beta} \sin \beta \theta \geq 0, \quad \forall \omega>0,
$$

which completes the proof. 
Remark 2.4. With the aid of Lemma 2.3, we can show that in the H-N model (2.7), if the initial electric and magnetic fields are divergence free $\left(\right.$ i.e. $\nabla \cdot \boldsymbol{E}_{0}=\nabla \cdot \boldsymbol{H}_{0}=0$ ), then we have $\nabla \cdot \boldsymbol{E}=\nabla \cdot \boldsymbol{H}=\nabla \cdot \boldsymbol{P}=0$ in $\Omega \times(0, T]$. Indeed, taking the divergence of two equations in (2.7a), we find readily that $\nabla \cdot \boldsymbol{H}=0$ and $\nabla \cdot\left(\epsilon_{\infty} \boldsymbol{E}+\boldsymbol{P}\right)=0$. Thus we derive from (2.12) and (2.14) that

$$
\epsilon_{\infty}{ }^{C}\left({ }_{0} \mathcal{D}_{t}^{\alpha}+1\right)^{\beta} \nabla \cdot \boldsymbol{P}=\epsilon_{\infty} \int_{0}^{t} e_{\alpha, 1-\alpha \beta}^{-\beta}(t-s ;-1) \partial_{s} \nabla \cdot \boldsymbol{P} \mathrm{d} s=-\Delta \epsilon \nabla \cdot \boldsymbol{P} .
$$

Testing (2.15) with $\partial_{t} \nabla \cdot \boldsymbol{P}$ leads to

$$
\epsilon_{\infty}\left({ }^{C}\left({ }_{0} \mathcal{D}_{t}^{\alpha}+1\right)^{\beta} \nabla \cdot \boldsymbol{P}(\cdot, t), \partial_{t} \nabla \cdot \boldsymbol{P}(\cdot, t)\right)+\Delta \epsilon\left(\nabla \cdot \boldsymbol{P}(\cdot, t), \partial_{t} \nabla \cdot \boldsymbol{P}(\cdot, t)\right)=0 .
$$

Integrating it with respect to $t$ from $t=0$ to any $T>0$, we deduce from (2.16) and Lemma 2.3 that the first term of the resulting equation is nonnegative, and $\|\nabla \cdot \boldsymbol{P}(\cdot, T)\| \leq 0$. Therefore, we can claim $\nabla \cdot \boldsymbol{P}=0$, so we can further derive $\epsilon_{\infty} \nabla \cdot \boldsymbol{E}=-\nabla \cdot \boldsymbol{P}=0$.

With Lemma 2.3 at our disposal, we can prove the following stability result for the H-N model (2.7).

Theorem 2.5. If $\boldsymbol{E}_{0}, \boldsymbol{H}_{0} \in \boldsymbol{L}^{2}(\Omega)$ in (2.7), then its solution $\boldsymbol{E}, \boldsymbol{H}, \boldsymbol{P} \in L^{\infty}\left(0, T ; \boldsymbol{L}^{2}(\Omega)\right)$ satisfying

$$
\mathscr{E}(t):=\epsilon_{\infty}\|\boldsymbol{E}(\cdot, t)\|^{2}+\|\boldsymbol{H}(\cdot, t)\|^{2} \leq \epsilon_{\infty}\left\|\boldsymbol{E}_{0}\right\|^{2}+\left\|\boldsymbol{H}_{0}\right\|^{2}:=\mathscr{E}_{0}, \quad \forall t \in(0, T),
$$

and

$$
\|\boldsymbol{P}\|_{L^{\infty}\left(0, T ; \boldsymbol{L}^{2}(\Omega)\right)} \leq \Delta \epsilon B\|\boldsymbol{E}\|_{L^{\infty}\left(0, T ; \boldsymbol{L}^{2}(\Omega)\right)},
$$

where the constant $B$ is given by

$$
B=T^{\alpha \beta} \sum_{k=0}^{\infty} \frac{\left|(\beta)_{k}\right|}{\alpha(k+\beta)|\Gamma(\alpha k+\alpha \beta)|} \frac{T^{\alpha k}}{k !} .
$$

Proof. Multiplying the first equation in (2.7a) by $\boldsymbol{E}$, and integrating the resulted equation by the Green's formula over $\Omega$, we obtain that

$$
\epsilon_{\infty}\left(\partial_{t} \boldsymbol{E}, \boldsymbol{E}\right)+\left(\partial_{t} \boldsymbol{P}, \boldsymbol{E}\right)-(\boldsymbol{H}, \nabla \times \boldsymbol{E})=0,
$$

where we used the boundary condition (2.7d). Similarly, we derive from the second equation in (2.7a) that

$$
\left(\partial_{t} \boldsymbol{H}, \boldsymbol{H}\right)+(\nabla \times \boldsymbol{E}, \boldsymbol{H})=0 .
$$

As a direct consequence of (2.19) and (2.20), we have

$$
\epsilon_{\infty}\left(\partial_{t} \boldsymbol{E}, \boldsymbol{E}\right)+\left(\partial_{t} \boldsymbol{H}, \boldsymbol{H}\right)+\left(\partial_{t} \boldsymbol{P}, \boldsymbol{E}\right)=0, \text { i.e. } \frac{1}{2} \mathscr{E}^{\prime}(t)=-\left(\partial_{t} \boldsymbol{P}, \boldsymbol{E}\right)
$$

In view of (2.14), we eliminate $\boldsymbol{E}$ and integrate the resulted equation with respect to $t$ over $(0, T)$, which, together with (2.12) and Lemma 2.3, leads to

$$
\begin{aligned}
\frac{1}{2} \mathscr{E}(T)-\frac{1}{2} \mathscr{E}_{0} & =-\frac{1}{\Delta \epsilon} \int_{0}^{T}\left(\partial_{t} \boldsymbol{P},{ }^{C}\left({ }_{0} \mathcal{D}_{t}^{\alpha}+1\right)^{\beta} \boldsymbol{P}\right) \mathrm{d} t \\
& =-\frac{1}{\Delta \epsilon} \int_{0}^{T} \int_{0}^{t} e_{\alpha, 1-\alpha \beta}^{-\beta}(t-s ;-1)\left(\partial_{t} \boldsymbol{P}, \partial_{s} \boldsymbol{P}\right) \mathrm{d} s \mathrm{~d} t \leq 0,
\end{aligned}
$$


where we recall that $\Delta \epsilon>0$. This yields $(2.17)$.

We now turn to (2.18). It is clear that by $(2.7 \mathrm{~b})$,

$$
\begin{aligned}
|\boldsymbol{P}(\boldsymbol{x}, t)| & \leq \Delta \epsilon \int_{0}^{t}\left|e_{\alpha, \alpha \beta}^{\beta}(t-s ;-1) \boldsymbol{E}(\boldsymbol{x}, s)\right| \mathrm{d} s \leq \Delta \epsilon \sup _{0 \leq s \leq t}|\boldsymbol{E}(\boldsymbol{x}, s)| \int_{0}^{t}\left|e_{\alpha, \alpha \beta}^{\beta}(t-s ;-1)\right| \mathrm{d} s \\
& \leq \Delta \epsilon \sup _{0 \leq s \leq t}|\boldsymbol{E}(\boldsymbol{x}, s)| \int_{0}^{T}\left|e_{\alpha, \alpha \beta}^{\beta}(u ;-1)\right| \mathrm{d} u .
\end{aligned}
$$

We derive from (2.8) and direct calculation that

$$
\int_{0}^{T}\left|e_{\alpha, \alpha \beta}^{\beta}(u ;-1)\right| \mathrm{d} u=\int_{0}^{T} u^{\alpha \beta-1}\left|E_{\alpha, \alpha \beta}^{\beta}(u ;-1)\right| \mathrm{d} u \leq \sum_{k=0}^{\infty} \frac{\left|(\beta)_{k}\right|}{\Gamma(\alpha k+\alpha \beta)} \frac{1}{k !} \int_{0}^{T} u^{\alpha k+\alpha \beta-1} \mathrm{~d} u:=B,
$$

where the quantity $B$ is finite ( $c f$. [24], Thm. 5 for the estimates of generalised ML functions). Thus, we obtain the first inequality but wish to show the second inequality below

$$
|\boldsymbol{P}(\boldsymbol{x}, t)|^{2} \leq(\Delta \epsilon)^{2} B^{2}\left(\sup _{0 \leq s \leq t}|\boldsymbol{E}(\boldsymbol{x}, s)|\right)^{2} \leq(\Delta \epsilon)^{2} B^{2} \sup _{0 \leq s \leq t}|\boldsymbol{E}(\boldsymbol{x}, s)|^{2} .
$$

Let $s_{0} \in[0, t]$ satisfy

$$
\left|\boldsymbol{E}\left(\boldsymbol{x}, s_{0}\right)\right|^{2}=\sup _{0 \leq s \leq t}|\boldsymbol{E}(\boldsymbol{x}, s)|^{2}, \quad \text { so }\left|\boldsymbol{E}\left(\boldsymbol{x}, s_{0}\right)\right| \geq|\boldsymbol{E}(\boldsymbol{x}, s)|, \quad \forall s \in[0, t],
$$

which implies

$$
\left|\boldsymbol{E}\left(\boldsymbol{x}, s_{0}\right)\right|^{2} \geq\left(\sup _{0 \leq s \leq t}|\boldsymbol{E}(\boldsymbol{x}, s)|\right)^{2},
$$

leading to the second inequality in (2.21). Therefore, we have

$$
\sup _{0 \leq s \leq t}|\boldsymbol{P}(\boldsymbol{x}, s)|^{2} \leq(\Delta \epsilon)^{2} B^{2} \sup _{0 \leq s \leq t}|\boldsymbol{E}(\boldsymbol{x}, s)|^{2}, \quad \forall t \in(0, T] .
$$

Integrating this inequality over $\Omega$, leads to (2.18).

Remark 2.6. It is known from standard analysis that the $L^{2}$-stability in Theorem 2.5 can ensure the uniqueness of the solution of the H-N model (2.7). In fact, we can follow the argument in Theorem 3.8 of [25] for the Drude model to show the existence of the solution. Here, we sketch the idea for the readers' reference. Let $\hat{f}(s)$ be the Laplace transform of $f(t), t \geq 0$. Then we can transform (2.7) into

$$
\epsilon_{\infty}\left(s \hat{\boldsymbol{E}}-\boldsymbol{E}_{0}\right)+s \hat{\boldsymbol{P}}=\nabla \times \hat{\boldsymbol{H}}, \quad s \hat{\boldsymbol{H}}-\boldsymbol{H}_{0}=-\nabla \times \hat{\boldsymbol{E}}, \quad \hat{\boldsymbol{P}}=\frac{\Delta \epsilon}{\left(1+s^{\alpha}\right)^{\beta}} \hat{\boldsymbol{E}} .
$$

Eliminating $\hat{\boldsymbol{H}}$ and $\hat{\boldsymbol{P}}$ from the first equation by other two equations, yields

$$
\left(\epsilon_{\infty}+\frac{\Delta \epsilon}{\left(1+s^{\alpha}\right)^{\beta}}\right) s^{2} \hat{\boldsymbol{E}}+\nabla \times \nabla \times \hat{\boldsymbol{E}}=\epsilon_{\infty} s \boldsymbol{E}_{0}+\nabla \times \boldsymbol{H}_{0} .
$$

A weak form is to find $\hat{\boldsymbol{E}} \in H_{0}(\operatorname{curl}, \Omega)$ such that

$$
\left(\epsilon_{\infty}+\frac{\Delta \epsilon}{\left(1+s^{\alpha}\right)^{\beta}}\right) s^{2}(\hat{\boldsymbol{E}}, \boldsymbol{\phi})+(\nabla \times \hat{\boldsymbol{E}}, \nabla \times \boldsymbol{\phi})=\left(\epsilon_{\infty} s \boldsymbol{E}_{0}+\nabla \times \boldsymbol{H}_{0}, \boldsymbol{\phi}\right), \quad \forall \boldsymbol{\phi} \in H_{0}(\operatorname{curl}, \Omega) .
$$

We infer from the Lax-Milgram lemma that for any $s>0$, it admits a unique solution $\hat{\boldsymbol{E}} \in H_{0}(\operatorname{curl}, \Omega)$, provided that $\boldsymbol{E}_{0}, \nabla \times \boldsymbol{H}_{0} \in \boldsymbol{L}^{2}(\Omega)(c f$. [7]). The inverse Laplace transform of $\hat{\boldsymbol{E}}$ is $\boldsymbol{E}$, and the uniqueness of $\boldsymbol{E} \in H_{0}(\operatorname{curl}, \Omega)$ follows from the uniqueness of the Laplace transform. Then we have the regularity of $\boldsymbol{P} \in H_{0}(\operatorname{curl}, \Omega)$ from the last equation of (2.22). The existence and uniqueness of $\boldsymbol{H} \in H(\operatorname{curl}, \Omega)$ can be assured by the same argument. 


\section{AnAlysis of A SEMi-DiscRete time-DisCRETiSATION SCHEME}

In this section, we propose a time-discretisation scheme for the $\mathrm{H}-\mathrm{N}$ model (2.7), and conduct the stability and convergence analysis.

\subsection{Time discretisation}

We start with a weak form of (2.7). Multiplying three equations in (2.7) by the respective test functions, integrating over $\Omega$ and using the boundary conditions, we follow the framework in [7], P. 18, 19 and arrive at the weak form, that is, to find $\boldsymbol{E} \in H_{0}(\operatorname{curl}, \Omega)$ and $\boldsymbol{H}, \boldsymbol{P} \in \boldsymbol{L}^{2}(\Omega)$ such that

$$
\begin{array}{ll}
\epsilon_{\infty}\left(\partial_{t} \boldsymbol{E}, \boldsymbol{\phi}\right)+\left(\partial_{t} \boldsymbol{P}, \boldsymbol{\phi}\right)-(\boldsymbol{H}, \nabla \times \boldsymbol{\phi})=0, & \forall \boldsymbol{\phi} \in H_{0}(\mathrm{curl}, \Omega), \\
\left(\partial_{t} \boldsymbol{H}, \boldsymbol{\psi}\right)+(\nabla \times \boldsymbol{E}, \boldsymbol{\psi})=0, & \forall \boldsymbol{\psi} \in \boldsymbol{L}^{2}(\Omega), \\
(\boldsymbol{P}, \boldsymbol{\varphi})=\Delta \epsilon \int_{0}^{t} e_{\alpha, \alpha \beta}^{\beta}(t-s ;-1)(\boldsymbol{E}(\cdot, s), \boldsymbol{\varphi}) \mathrm{d} s, & \forall \boldsymbol{\varphi} \in \boldsymbol{L}^{2}(\Omega) .
\end{array}
$$

Note that the result in Remark 2.6 (based on the argument in [25], Thm. 3.8) carries over to this problem with a suitable regularity assumption on the initial fields $\boldsymbol{E}_{0}, \boldsymbol{H}_{0}$.

We partition the time interval $[0, T]$, and denote

$$
t_{k}=k \Delta t, \quad k=0,1, \cdots, N_{t}, \quad \Delta t=T / N_{t} ; \quad \delta_{t} u^{k}=\frac{u^{k}-u^{k-1}}{\Delta t},
$$

where $u^{k}$ stands for the approximation of $u$ at time $t_{k}$.

We first consider the time discretisation of $(2.7 \mathrm{~b})$, and employ the piecewise constant approximation $I_{\Delta t} \boldsymbol{E}$ of $\boldsymbol{E}$ :

$$
\begin{aligned}
\boldsymbol{P}\left(\boldsymbol{x}, t_{k}\right) & =\Delta \epsilon \int_{0}^{t_{k}} e_{\alpha, \alpha \beta}^{\beta}\left(t_{k}-s ;-1\right) I_{\Delta t} \boldsymbol{E}(\boldsymbol{x}, s) \mathrm{d} s+\Delta \epsilon \boldsymbol{R}_{0}^{k}(\boldsymbol{x}) \\
& =\Delta \epsilon \sum_{j=1}^{k}\left(\int_{t_{j-1}}^{t_{j}} e_{\alpha, \alpha \beta}^{\beta}\left(t_{k}-s ;-1\right) \mathrm{d} s\right) \boldsymbol{E}\left(\boldsymbol{x}, t_{j}\right)+\Delta \epsilon \boldsymbol{R}_{0}^{k}(\boldsymbol{x}) \\
& =\Delta \epsilon \sum_{j=1}^{k} \varpi_{k-j}^{(\alpha, \beta)} \boldsymbol{E}\left(\boldsymbol{x}, t_{j}\right)+\Delta \epsilon \boldsymbol{R}_{0}^{k}(\boldsymbol{x}), \quad k \geq 1,
\end{aligned}
$$

where the residual and the weights are given by

$$
\begin{aligned}
\boldsymbol{R}_{0}^{k}(\boldsymbol{x}) & :=\int_{0}^{t_{k}} e_{\alpha, \alpha \beta}^{\beta}\left(t_{k}-s ;-1\right)\left(\boldsymbol{E}(\boldsymbol{x}, s)-I_{\Delta t} \boldsymbol{E}(\boldsymbol{x}, s)\right) \mathrm{d} s \\
& =\sum_{j=1}^{k} \int_{t_{j-1}}^{t_{j}} e_{\alpha, \alpha \beta}^{\beta}\left(t_{k}-s ;-1\right)\left(\boldsymbol{E}(\boldsymbol{x}, s)-\boldsymbol{E}\left(\boldsymbol{x}, t_{j}\right)\right) \mathrm{d} s,
\end{aligned}
$$

and

$$
\varpi_{k-j}^{(\alpha, \beta)}:=\int_{t_{j-1}}^{t_{j}} e_{\alpha, \alpha \beta}^{\beta}\left(t_{k}-s ;-1\right) \mathrm{d} s=\int_{(k-j) \Delta t}^{(k-j+1) \Delta t} e_{\alpha, \alpha \beta}^{\beta}(s ;-1) \mathrm{d} s,
$$

respectively. By (A.2), we can rewrite the weights as

$$
\varpi_{k-j}^{(\alpha, \beta)}=e_{\alpha, \alpha \beta+1}^{\beta}((k-j+1) \Delta t ;-1)-e_{\alpha, \alpha \beta+1}^{\beta}((k-j) \Delta t ;-1), \quad 1 \leq j \leq k .
$$

Note that we can compute them accurately by using the codes in [14] for the ML functions.

We have the following important property of the weights in (3.5). 
Lemma 3.1. For $0<\alpha<1,0<\beta \leq 1$, and $1 \leq k \leq N_{t}$, the weights $\left\{\varpi_{k-j}^{(\alpha, \beta)}\right\}_{j=1}^{k}$ satisfy

$$
0 \leq \varpi_{k-1}^{(\alpha, \beta)} \leq \varpi_{k-2}^{(\alpha, \beta)} \leq \cdots \leq \varpi_{1}^{(\alpha, \beta)} \leq \varpi_{0}^{(\alpha, \beta)}=(\Delta t)^{\alpha \beta} E_{\alpha, \alpha \beta+1}^{\beta}\left(-(\Delta t)^{\alpha}\right)
$$

and $\varpi_{0}^{(\alpha, \beta)}$ is finite.

Proof. Using the integral mean-value theorem, we find from (3.4) that

$$
\varpi_{k-j}^{(\alpha, \beta)}=\int_{t_{j-1}}^{t_{j}} e_{\alpha, \alpha \beta}^{\beta}\left(t_{k}-s ;-1\right) \mathrm{d} s=\Delta t e_{\alpha, \alpha \beta}^{\beta}\left(t_{k}-\theta ;-1\right), \quad \exists \theta \in\left(t_{j-1}, t_{j}\right), \quad 1 \leq j \leq k .
$$

It is nonnegative and decreasing, since $e_{\alpha, \alpha \beta}^{\beta}(z ;-1)$ is completely monotonic for $0<\alpha<1,0<\beta \leq 1$, and $z>0$ (see (A.4)). Therefore, we have the monotonicity of the discrete kernels and $\varpi_{k-1}^{(\alpha, \beta)} \geq 0\left(1 \leq k \leq N_{t}\right)$. By virtue of (3.5), we have $\varpi_{0}^{(\alpha, \beta)}=(\Delta t)^{\alpha \beta} E_{\alpha, \alpha \beta+1}^{\beta}\left(-(\Delta t)^{\alpha}\right)$, which is finite due to (A.3).

Remark 3.2. In what follows, we shall not consider the D-C model (i.e. $\alpha=1$ ). In fact, the computational codes for the ML functions in [14] excludes the case with $\alpha=1$. In fact, the D-C model can be solved by a very different method which we plan to report in a separate future work.

Now we present the semi-discrete time-discretisation scheme for the H-N model (2.7): find $\boldsymbol{E}^{k} \in H_{0}(\operatorname{curl}, \Omega)$ and $\boldsymbol{H}^{k}, \boldsymbol{P}^{k} \in \boldsymbol{L}^{2}(\Omega)$ such that

$$
\begin{array}{ll}
\epsilon_{\infty}\left(\delta_{t} \boldsymbol{E}^{k}, \boldsymbol{\phi}\right)+\left(\delta_{t} \boldsymbol{P}^{k}, \boldsymbol{\phi}\right)-\left(\boldsymbol{H}^{k}, \nabla \times \boldsymbol{\phi}\right)=0, & \forall \boldsymbol{\phi} \in H_{0}(\operatorname{curl}, \Omega), \\
\left(\delta_{t} \boldsymbol{H}^{k}, \boldsymbol{\psi}\right)+\left(\nabla \times \boldsymbol{E}^{k}, \boldsymbol{\psi}\right)=0, & \forall \boldsymbol{\psi} \in \boldsymbol{L}^{2}(\Omega), \\
\left(\boldsymbol{P}^{k}, \boldsymbol{\varphi}\right)=\Delta \epsilon \sum_{j=1}^{k} \varpi_{k-j}^{(\alpha, \beta)}\left(\boldsymbol{E}^{j}, \boldsymbol{\varphi}\right), & \forall \boldsymbol{\varphi} \in \boldsymbol{L}^{2}(\Omega),
\end{array}
$$

for $k=1,2, \cdots, N_{t}$, where $\boldsymbol{E}^{0}=\boldsymbol{E}_{0}(\boldsymbol{x}), \boldsymbol{H}^{0}=\boldsymbol{H}_{0}(\boldsymbol{x})$ and $\boldsymbol{P}^{0}=\mathbf{0}$.

Remark 3.3. Following the derivation in Remark 2.4, we can show $\nabla \times \boldsymbol{H}^{k}=0$ and

$$
\nabla \times\left(\epsilon_{\infty} \boldsymbol{E}^{k}+\boldsymbol{P}^{k}\right)=0
$$

provided that the initial fields $\boldsymbol{E}^{0}$ and $\boldsymbol{H}^{0}$ are divergence free. With this, we can recursively prove that $\nabla \times \boldsymbol{E}^{k}=$ 0 , so is $\boldsymbol{P}^{k}$. Indeed, when $k=1$, substituting (3.6c) into (3.7) and using Lemma 2.3, we find $\nabla \times \boldsymbol{E}^{1}=\nabla \times \boldsymbol{P}^{1}=0$. Similarly, we are able to show the result for $k=2$. Recursively, we deduce this property for general $k \geq 3$.

\subsection{Stability and discrete energy dissipation}

In the convergence analysis, it is necessary to consider a more general setting:

$$
\begin{array}{ll}
\epsilon_{\infty}\left(\delta_{t} \boldsymbol{E}^{k}, \boldsymbol{\phi}\right)+\left(\delta_{t} \boldsymbol{P}^{k}, \boldsymbol{\phi}\right)-\left(\boldsymbol{H}^{k}, \nabla \times \boldsymbol{\phi}\right)=\left(\boldsymbol{f}^{k}, \boldsymbol{\phi}\right), & \forall \boldsymbol{\phi} \in H_{0}(\operatorname{curl}, \Omega), \\
\left(\delta_{t} \boldsymbol{H}^{k}, \boldsymbol{\psi}\right)+\left(\nabla \times \boldsymbol{E}^{k}, \boldsymbol{\psi}\right)=\left(\boldsymbol{g}^{k}, \boldsymbol{\psi}\right), & \forall \boldsymbol{\psi} \in \boldsymbol{L}^{2}(\Omega), \\
\left(\boldsymbol{P}^{k}, \boldsymbol{\varphi}\right)=\Delta \epsilon \sum_{j=1}^{k} \varpi_{k-j}^{(\alpha, \beta)}\left(\boldsymbol{E}^{j}, \boldsymbol{\varphi}\right)+\Delta \epsilon\left(\boldsymbol{h}^{k}, \boldsymbol{\varphi}\right), & \forall \boldsymbol{\varphi} \in \boldsymbol{L}^{2}(\Omega),
\end{array}
$$

where $\boldsymbol{E}^{0}=\boldsymbol{E}_{0}(\boldsymbol{x}), \boldsymbol{H}^{0}=\boldsymbol{H}_{0}(\boldsymbol{x})$ and $\boldsymbol{P}^{0}=\mathbf{0}$. We shall see from the error equations (3.26) and (3.27) for convergence analysis that these non-homogeneous data will correspond to the time-discretisation errors of the fields. 
Theorem 3.4. Let $\boldsymbol{E}^{k}, \boldsymbol{P}^{k}, \boldsymbol{H}^{k}$ be the solutions of (3.6) or (3.8), and define

$$
\mathscr{E}^{k}:=\epsilon_{\infty}\left\|\boldsymbol{E}^{k}\right\|^{2}+\left\|\boldsymbol{H}^{k}\right\|^{2}+\Delta \epsilon \sum_{j=1}^{k} \varpi_{k-j}^{(\alpha, \beta)}\left\|\boldsymbol{E}^{j}\right\|^{2}, \quad k \geq 1 ; \quad \mathscr{E}^{0}:=\epsilon_{\infty}\left\|\boldsymbol{E}^{0}\right\|^{2}+\left\|\boldsymbol{H}^{0}\right\|^{2} .
$$

Then the scheme (3.6) is unconditionally stable in the sense that for all $\Delta t>0$,

$$
\mathscr{E}^{k} \leq \mathscr{E}^{k-1} \leq \cdots \leq \mathscr{E}^{1} \leq \mathscr{E}^{0}
$$

For the scheme (3.8) with nonhomogeneous data, if $\varrho \Delta t<1$ for some given positive constant $\varrho>0$, and

$$
\boldsymbol{Q}^{k}:=\Delta t \sum_{j=1}^{k}\left(\left\|\boldsymbol{f}^{j}\right\|^{2}+(\Delta \epsilon)^{2}\left\|\delta_{t} \boldsymbol{h}^{j}\right\|^{2}+\left\|\boldsymbol{g}^{j}\right\|^{2}\right)<\infty
$$

then we have

$$
\mathscr{E}^{k} \leq \frac{1}{1-\varrho \Delta t} \exp \left(\frac{\varrho t_{k-1}}{1-\varrho \Delta t}\right)\left(\mathscr{E}^{0}+\frac{1}{\varrho} \boldsymbol{Q}^{k}\right), \quad k \geq 1 .
$$

Proof. We first prove (3.12), and then (3.10) follows straightforwardly.

Taking $\boldsymbol{\phi}=\Delta t \boldsymbol{E}^{k}$ in (3.8a) and $\boldsymbol{\psi}=\Delta t \boldsymbol{H}^{k}$ in (3.8b), respectively, and adding two resulted equations together, we obtain

$$
\begin{gathered}
\epsilon_{\infty}\left(\boldsymbol{E}^{k}-\boldsymbol{E}^{k-1}, \boldsymbol{E}^{k}\right)+\left(\boldsymbol{H}^{k}-\boldsymbol{H}^{k-1}, \boldsymbol{H}^{k}\right)+\left(\boldsymbol{P}^{k}-\boldsymbol{P}^{k-1}, \boldsymbol{E}^{k}\right) \\
=\Delta t\left(\boldsymbol{f}^{k}, \boldsymbol{E}^{k}\right)+\Delta t\left(\boldsymbol{g}^{k}, \boldsymbol{H}^{k}\right) .
\end{gathered}
$$

We eliminate $\boldsymbol{P}$ from the above identity by using (3.8c) with $\boldsymbol{\varphi}=\boldsymbol{E}^{k}$, so we can rewrite (3.13) as

$$
\begin{aligned}
\epsilon_{\infty}\left(\boldsymbol{E}^{k}, \boldsymbol{E}^{k}\right)+ & \left(\boldsymbol{H}^{k}, \boldsymbol{H}^{k}\right)+\Delta \epsilon \sum_{j=1}^{k} \varpi_{k-j}^{(\alpha, \beta)}\left(\boldsymbol{E}^{j}, \boldsymbol{E}^{k}\right) \\
= & \epsilon_{\infty}\left(\boldsymbol{E}^{k-1}, \boldsymbol{E}^{k}\right)+\left(\boldsymbol{H}^{k-1}, \boldsymbol{H}^{k}\right)+\Delta \epsilon \sum_{j=1}^{k-1} \varpi_{k-1-j}^{(\alpha, \beta)}\left(\boldsymbol{E}^{j}, \boldsymbol{E}^{k}\right) \\
& +\Delta t\left(\boldsymbol{f}^{k}+\Delta \epsilon \delta_{t} \boldsymbol{h}^{k}, \boldsymbol{E}^{k}\right)+\Delta t\left(\boldsymbol{g}^{k}, \boldsymbol{H}^{k}\right) .
\end{aligned}
$$

Rearranging (3.14) yields

$$
\begin{aligned}
\left(\epsilon_{\infty}+\Delta \epsilon \varpi_{0}^{(\alpha, \beta)}\right)\left\|\boldsymbol{E}^{k}\right\|^{2}+\left\|\boldsymbol{H}^{k}\right\|^{2} \\
=\epsilon_{\infty}\left(\boldsymbol{E}^{k-1}, \boldsymbol{E}^{k}\right)+\left(\boldsymbol{H}^{k-1}, \boldsymbol{H}^{k}\right)+\Delta \epsilon \sum_{j=1}^{k-1}\left(\varpi_{k-1-j}^{(\alpha, \beta)}-\varpi_{k-j}^{(\alpha, \beta)}\right)\left(\boldsymbol{E}^{j}, \boldsymbol{E}^{k}\right) \\
\quad+\Delta t\left(\boldsymbol{f}^{k}+\Delta \epsilon \delta_{t} \boldsymbol{h}^{k}, \boldsymbol{E}^{k}\right)+\Delta t\left(\boldsymbol{g}^{k}, \boldsymbol{H}^{k}\right) .
\end{aligned}
$$

For $k \geq 2$, using the Cauchy-Schwarz inequality and Lemma 3.1, we obtain

$$
\begin{aligned}
\left(\epsilon_{\infty}+\Delta \epsilon \varpi_{0}^{(\alpha, \beta)}\right)\left\|\boldsymbol{E}^{k}\right\|^{2}+\left\|\boldsymbol{H}^{k}\right\|^{2} \leq & \frac{\epsilon_{\infty}}{2}\left(\left\|\boldsymbol{E}^{k}\right\|^{2}+\left\|\boldsymbol{E}^{k-1}\right\|^{2}\right)+\frac{1}{2}\left(\left\|\boldsymbol{H}^{k}\right\|^{2}+\left\|\boldsymbol{H}^{k-1}\right\|^{2}\right) \\
& +\frac{\Delta \epsilon}{2} \sum_{j=1}^{k-1}\left(\varpi_{k-1-j}^{(\alpha, \beta)}-\varpi_{k-j}^{(\alpha, \beta)}\right)\left(\left\|\boldsymbol{E}^{k}\right\|^{2}+\left\|\boldsymbol{E}^{j}\right\|^{2}\right) \\
& +\frac{\varrho \Delta t}{2}\left(\left\|\boldsymbol{E}^{k}\right\|^{2}+\left\|\boldsymbol{H}^{k}\right\|^{2}\right)+\frac{\Delta t}{2 \varrho}\left(\left\|\boldsymbol{f}^{k}+\Delta \epsilon \delta_{t} \boldsymbol{h}^{k}\right\|^{2}+\left\|\boldsymbol{g}^{k}\right\|^{2}\right),
\end{aligned}
$$


where $\varrho>0$ is a constant independent of $\Delta t$ and $k$. It is evident that

$$
\sum_{j=1}^{k-1}\left(\varpi_{k-1-j}^{(\alpha, \beta)}-\varpi_{k-j}^{(\alpha, \beta)}\right)\left\|\boldsymbol{E}^{k}\right\|^{2}=\left(\varpi_{0}^{(\alpha, \beta)}-\varpi_{k-1}^{(\alpha, \beta)}\right)\left\|\boldsymbol{E}^{k}\right\|^{2} .
$$

Consequently, by (3.9), we find from (3.16) and (3.17) immediately that

$$
\mathscr{E}^{k}-\varrho \Delta t\left(\left\|\boldsymbol{E}^{k}\right\|^{2}+\left\|\boldsymbol{H}^{k}\right\|^{2}\right) \leq \mathscr{E}^{k-1}+\frac{\Delta t}{\varrho}\left(\left\|\boldsymbol{f}^{k}+\Delta \epsilon \delta_{t} \boldsymbol{h}^{k}\right\|^{2}+\left\|\boldsymbol{g}^{k}\right\|^{2}\right),
$$

which implies

$$
(1-\varrho \Delta t) \mathscr{E}^{k} \leq \mathscr{E}^{k-1}+\frac{\Delta t}{\varrho}\left(\left\|\boldsymbol{f}^{k}\right\|^{2}+(\Delta \epsilon)^{2}\left\|\delta_{t} \boldsymbol{h}^{k}\right\|^{2}+\left\|\boldsymbol{g}^{k}\right\|^{2}\right) .
$$

In fact, (3.19) also holds for $k=1$. Indeed, by (3.13) with $k=1$, and understanding the summation $\sum_{n=1}^{0}=0$ in (3.14)-(3.17), we can get (3.19) with $k=1$ readily.

For clarity, we set $k=j$ and rewrite (3.19) as

$$
(1-\varrho \Delta t)\left(\mathscr{E}^{j}-\mathscr{E}^{j-1}\right) \leq \varrho \Delta t \mathscr{E}^{j-1}+\frac{\Delta t}{\varrho}\left(\left\|\boldsymbol{f}^{j}\right\|^{2}+(\Delta \epsilon)^{2}\left\|\delta_{t} \boldsymbol{h}^{j}\right\|^{2}+\left\|\boldsymbol{g}^{j}\right\|^{2}\right), \quad j \geq 1 .
$$

Summing it up for $1 \leq j \leq k$, we have that if $\varrho \Delta t<1$,

$$
\mathscr{E}^{k} \leq \frac{1}{1-\varrho \Delta t}\left\{\varrho \Delta t \sum_{j=1}^{k-1} \mathscr{E}^{j}+\mathscr{E}^{0}+\frac{\Delta t}{\varrho} \sum_{j=1}^{k}\left(\left\|\boldsymbol{f}^{j}\right\|^{2}+(\Delta \epsilon)^{2}\left\|\delta_{t} \boldsymbol{h}^{j}\right\|^{2}+\left\|\boldsymbol{g}^{j}\right\|^{2}\right)\right\} .
$$

Using the discrete Grönwall's inequality (see, e.g. [36], Lem. 1.4.2), we derive (3.12) directly.

We now turn to (3.6). In this case, we have $\boldsymbol{f}^{k}=\boldsymbol{g}^{k}=\boldsymbol{h}^{k}=\mathbf{0}$ in (3.13). Following the same lines as in the above derivations, we find readily that (3.18) becomes $\mathscr{E}^{k} \leq \mathscr{E}^{k-1}$, so the decay of the discrete energy in (3.10) holds. The proof is completed.

Remark 3.5. We can represent the constant in the bound (3.12) more explicitly so that it does not depend on $\Delta t$. For example, we take $\varrho=1$ and assume that $1-\Delta t \geq c_{*}>0$, i.e. $\Delta t<1-c^{*}$. Then

$$
\mathscr{E}^{k} \leq \frac{1}{c_{*}} \exp \left(\frac{t_{k-1}}{c_{*}}\right)\left(\mathscr{E}^{0}+\boldsymbol{Q}^{k}\right), \quad k \geq 1 .
$$

With the aid of Theorem 3.4, we can further derive the following bound for $\boldsymbol{P}^{k}$ in (3.6c).

Corollary 3.6. Let $\boldsymbol{E}^{k}, \boldsymbol{P}^{k}, \boldsymbol{H}^{k}$ be the solution of (3.6). Then we have

$$
\left\|\boldsymbol{P}^{k}\right\| \leq\left(\Delta \epsilon e_{\alpha, \alpha \beta+1}^{\beta}\left(t_{k} ;-1\right)\right) \max _{1 \leq j \leq k}\left\|\boldsymbol{E}^{j}\right\| \leq\left(\Delta \epsilon e_{\alpha, \alpha \beta+1}^{\beta}\left(t_{k} ;-1\right)\right) \sqrt{\mathscr{E} 0},
$$

for $k \geq 1$, where $e_{\alpha, \alpha \beta+1}^{\beta}\left(t_{k} ;-1\right)$ defined in $(2.8)$ is finite for $0<t_{k} \leq T$.

Proof. From (3.6c), we obtain

$$
\left\|\boldsymbol{P}^{k}\right\|^{2}=\left(\boldsymbol{P}^{k}, \boldsymbol{P}^{k}\right)=\Delta \epsilon \sum_{j=1}^{k} \varpi_{k-j}^{(\alpha, \beta)}\left(\boldsymbol{E}^{j}, \boldsymbol{P}^{k}\right) \leq \Delta \epsilon \sum_{j=1}^{k} \varpi_{k-j}^{(\alpha, \beta)}\left\|\boldsymbol{E}^{j}\right\|\left\|\boldsymbol{P}^{k}\right\| .
$$


Thus, one has

$$
\begin{aligned}
\left\|\boldsymbol{P}^{k}\right\| & \leq \Delta \epsilon \sum_{j=1}^{k} \varpi_{k-j}^{(\alpha, \beta)}\left\|\boldsymbol{E}^{j}\right\| \leq \Delta \epsilon\left(\max _{1 \leq j \leq k}\left\|\boldsymbol{E}^{j}\right\|\right)\left(\sum_{j=1}^{k} \varpi_{k-j}^{(\alpha, \beta)}\right) \\
& \leq \Delta \epsilon t_{k}^{\alpha \beta} E_{\alpha, \alpha \beta+1}^{\beta}\left(-t_{k}^{\alpha}\right)\left(\max _{1 \leq j \leq k}\left\|\boldsymbol{E}^{j}\right\|\right),
\end{aligned}
$$

where we used (3.5) and (A.3) to arrive at

$$
\sum_{j=1}^{k} \varpi_{k-j}^{(\alpha, \beta)}=e_{\alpha, \alpha \beta+1}^{\beta}\left(t_{k} ;-1\right)-e_{\alpha, \alpha \beta+1}^{\beta}(0 ;-1)=t_{k}^{\alpha \beta} E_{\alpha, \alpha \beta+1}^{\beta}\left(-t_{k}^{\alpha}\right)=e_{\alpha, \alpha \beta+1}^{\beta}\left(t_{k} ;-1\right) .
$$

Then we obtain the second inequality in (3.23) from (3.10) immediately. The proof is completed.

Remark 3.7. For the C-C model (i.e. $\beta=1$ ), the energy dissipation was proved by Li et al. [26], where the equation of the induced polarization and electric field was formulated as the Caputo fractional differential form (see $(2.14)$ with $\beta=1$ ). However, it appeared nontrivial to show the strong energy dissipation and stability similar to (3.10), as the bound therein contained a constant $C>1$ between $k$ th and $(k-1)$ th steps (see [26], Thm. 3.1). Though our result does not resolve this deficiency, as we work with the integral formulation of the induced polarization and electric field, and the discretisation schemes are different, we believe our argument can shed light on the analysis of the scheme based on the fractional differential form.

\subsection{Convergence analysis}

Next, we carry out the convergence analysis of the semi-discrete scheme (3.6). Denote $\boldsymbol{\varepsilon}_{1}^{k}=\boldsymbol{E}^{k}-\boldsymbol{E}\left(t_{k}\right)$, $\varepsilon_{2}^{k}=\boldsymbol{H}^{k}-\boldsymbol{H}\left(t_{k}\right)$, and $\varepsilon_{3}^{k}=\boldsymbol{P}^{k}-\boldsymbol{P}\left(t_{k}\right)$. Then we can derive the following error equations from subtracting (3.1) from (3.6):

$$
\begin{array}{ll}
\epsilon_{\infty}\left(\delta_{t} \varepsilon_{1}^{k}, \boldsymbol{\phi}\right)+\left(\delta_{t} \varepsilon_{3}^{k}, \boldsymbol{\phi}\right)-\left(\varepsilon_{2}^{k}, \nabla \times \boldsymbol{\phi}\right)=\left(\boldsymbol{R}_{1}^{k}, \boldsymbol{\phi}\right), & \forall \boldsymbol{\phi} \in H_{0}(\mathrm{curl}, \Omega), \\
\left(\delta_{t} \varepsilon_{2}^{k}, \boldsymbol{\psi}\right)+\left(\nabla \times \varepsilon_{1}^{k}, \boldsymbol{\psi}\right)=\left(\boldsymbol{R}_{2}^{k}, \boldsymbol{\phi}\right), & \forall \boldsymbol{\psi} \in \boldsymbol{L}^{2}(\Omega), \\
\left(\varepsilon_{3}^{k}, \boldsymbol{\varphi}\right)=\Delta \epsilon \sum_{j=1}^{k} \varpi_{k-j}^{(\alpha, \beta)}\left(\varepsilon_{1}^{j}, \boldsymbol{\varphi}\right)-\Delta \epsilon\left(\boldsymbol{R}_{0}^{k}, \boldsymbol{\varphi}\right), & \forall \boldsymbol{\varphi} \in \boldsymbol{L}^{2}(\Omega),
\end{array}
$$

for $k=1,2, \cdots, N_{t}$, where $\boldsymbol{R}_{0}^{k}$ is defined in (3.3) and

$$
\begin{aligned}
& \boldsymbol{R}_{1}^{k}(\boldsymbol{x})=\epsilon_{\infty}\left(\partial_{t} \boldsymbol{E}\left(\boldsymbol{x}, t_{k}\right)-\delta_{t} \boldsymbol{E}\left(\boldsymbol{x}, t_{k}\right)\right)+\partial_{t} \boldsymbol{P}\left(\boldsymbol{x}, t_{k}\right)-\delta_{t} \boldsymbol{P}\left(\boldsymbol{x}, t_{k}\right), \\
& \boldsymbol{R}_{2}^{k}(\boldsymbol{x})=\partial_{t} \boldsymbol{H}\left(\boldsymbol{x}, t_{k}\right)-\delta_{t} \boldsymbol{H}\left(\boldsymbol{x}, t_{k}\right) .
\end{aligned}
$$

Now, we can present the following convergence result for the semi-discrete scheme. Before the proof, we first give the following lemma.

Lemma 3.8. If $\boldsymbol{E}, \boldsymbol{P}, \boldsymbol{H} \in H^{2}\left(0, T ; \boldsymbol{L}^{2}(\Omega)\right)$ and $k \geq 1$, then we have

$$
\left\|\boldsymbol{R}_{1}^{k}\right\| \leq C \Delta t\left(\|\boldsymbol{E}\|_{H^{2}\left(0, T ; \boldsymbol{L}^{2}(\Omega)\right)}+\|\boldsymbol{P}\|_{H^{2}\left(0, T ; \boldsymbol{L}^{2}(\Omega)\right)}\right) ; \quad\left\|\boldsymbol{R}_{2}^{k}\right\| \leq C \Delta t\|\boldsymbol{H}\|_{H^{2}\left(0, T ; \boldsymbol{L}^{2}(\Omega)\right)},
$$

and

$$
\left\|\boldsymbol{R}_{0}^{k}\right\| \leq C \Delta t\|\boldsymbol{E}\|_{H^{1}\left(0, T ; \boldsymbol{L}^{2}(\Omega)\right)}, \quad\left\|\delta_{t} \boldsymbol{R}_{0}^{k}\right\| \leq C \Delta t\|\boldsymbol{E}\|_{H^{2}\left(0, T ; \boldsymbol{L}^{2}(\Omega)\right)},
$$

where $C$ is a generic positive constant independent of $\Delta t$ and any field but depending on $t_{k-1}$. 
Proof. From (3.27), we can obtain readily the estimates (3.28) using the standard finite difference analysis for $1 \leq k \leq N_{t}$.

We now derive (3.29). Denote $\boldsymbol{r}^{k}=\boldsymbol{E}(\boldsymbol{x}, t)-\boldsymbol{E}\left(\boldsymbol{x}, t_{k}\right), t \in\left(t_{k-1}, t_{k}\right)$. Then by (3.3), (A.2) and the completely monotonicity of $e_{\alpha, \alpha \beta}^{\beta}\left(z ;-(\Delta t)^{\alpha}\right)(0<\alpha<1,0<\beta \leq 1, z>0)$ (see (A.4)), we have

$$
\left\|\boldsymbol{R}_{0}^{k}\right\| \leq \Delta t\|\boldsymbol{E}\|_{H^{1}\left((0, T) ; \boldsymbol{L}^{2}(\Omega)\right)} \int_{0}^{t_{j}} e_{\alpha, \alpha \beta}^{\beta}\left(t_{j}-s ;-1\right) \mathrm{d} s=\Delta t e_{\alpha, \alpha \beta+1}^{\beta}\left(t_{j} ;-1\right)\|\boldsymbol{E}\|_{H^{1}\left(0, T ; \boldsymbol{L}^{2}(\Omega)\right)},
$$

and

$$
\begin{aligned}
\left\|\delta_{t} \boldsymbol{R}_{0}^{k}\right\|= & \frac{1}{\Delta t}\left\|\sum_{j=1}^{k} \int_{t_{j-1}}^{t_{j}} e_{\alpha, \alpha \beta}^{\beta}\left(t_{k}-s ;-1\right) \boldsymbol{r}^{j}(s) \mathrm{d} s-\sum_{j=1}^{k-1} \int_{t_{j-1}}^{t_{j}} e_{\alpha, \alpha \beta}^{\beta}\left(t_{k-1}-s ;-1\right) \boldsymbol{r}^{j}(s) \mathrm{d} s\right\| \\
\leq & \frac{1}{\Delta t}\left\|\sum_{j=1}^{k-1} \int_{t_{j-1}}^{t_{j}} e_{\alpha, \alpha \beta}^{\beta}\left(t_{k-1}-s ;-1\right)\left[\boldsymbol{r}^{j}(s+\Delta t)-\boldsymbol{r}^{j}(s)\right] \mathrm{d} s\right\|+\frac{1}{\Delta t}\left\|\int_{0}^{t_{1}} e_{\alpha, \alpha \beta}^{\beta}\left(t_{k}-s ;-1\right) \boldsymbol{r}^{1}(s) \mathrm{d} s\right\| \\
\leq & \frac{1}{\Delta t}\left(C(\Delta t)^{2}\|\boldsymbol{E}\|_{H^{2}\left(0, T ; \boldsymbol{L}^{2}(\Omega)\right)} \int_{0}^{t_{k-1}} e_{\alpha, \alpha \beta}^{\beta}\left(t_{k-1}-s ;-1\right) \mathrm{d} s\right. \\
& \left.+C \Delta t\|\boldsymbol{E}\|_{H^{1}\left(0, T ; \boldsymbol{L}^{2}(\Omega)\right)} \int_{0}^{\Delta t} e_{\alpha, \alpha \beta}^{\beta}\left(t_{k}-s ;-1\right) \mathrm{d} s\right) \\
\leq & C \Delta t\|\boldsymbol{E}\|_{H^{2}\left(0, T ; \boldsymbol{L}^{2}(\Omega)\right)} \int_{0}^{t_{k-1}} e_{\alpha, \alpha \beta}^{\beta}\left(t_{k-1}-s ;-1\right) \mathrm{d} s+C e_{\alpha, \alpha \beta}^{\beta}\left(t_{k-1} ;-1\right)\|\boldsymbol{E}\|_{H^{1}\left(0, T ; \boldsymbol{L}^{2}(\Omega)\right)} \int_{0}^{\Delta t} \mathrm{~d} s \\
\leq & C \Delta t\left(e_{\alpha, \alpha \beta+1}^{\beta}\left(t_{k-1} ;-1\right)\|\boldsymbol{E}\|_{H^{2}\left(0, T ; \boldsymbol{L}^{2}(\Omega)\right)}+e_{\alpha, \alpha \beta}^{\beta}\left(t_{k-1} ;-1\right)\|\boldsymbol{E}\|_{H^{1}\left(0, T ; \boldsymbol{L}^{2}(\Omega)\right)}\right) .
\end{aligned}
$$

Then by (A.3), the proof is completed.

In light of Theorem 3.4 and Lemma 3.8, we obtain the following convergence result on the semi-discrete scheme (3.6).

Theorem 3.9. Let $\boldsymbol{E}(\boldsymbol{x}, t), \boldsymbol{P}(\boldsymbol{x}, t), \boldsymbol{H}(\boldsymbol{x}, t)$ be the solution of $(2.7)$, and let $\boldsymbol{E}^{k}, \boldsymbol{P}^{k}, \boldsymbol{H}^{k}$ be the solution of (3.6). Assume that

$$
\boldsymbol{E} \in H^{2}\left(0, T ; H_{0}(\operatorname{curl}, \Omega) \cap \boldsymbol{L}^{2}(\Omega)\right) \text { and } \boldsymbol{P}, \boldsymbol{H} \in H^{2}\left(0, T ; \boldsymbol{L}^{2}(\Omega)\right) .
$$

Then for $\Delta t<1-c^{*}$ for given constant $c^{*} \in(0,1)$ as in Remark 3.5, we have the error estimate

$$
\begin{aligned}
& \left\|\boldsymbol{E}^{k}(\cdot)-\boldsymbol{E}\left(\cdot, t_{k}\right)\right\|^{2}+\left\|\boldsymbol{H}^{k}(\cdot)-\boldsymbol{H}\left(\cdot, t_{k}\right)\right\|^{2}+\left\|\boldsymbol{P}^{k}(\cdot)-\boldsymbol{P}\left(\cdot, t_{k}\right)\right\|^{2} \\
& \quad \leq C(\Delta t)^{2}\left(\|\boldsymbol{E}\|_{H^{2}\left(0, T ; \boldsymbol{L}^{2}(\Omega)\right)}^{2}+\|\boldsymbol{P}\|_{H^{2}\left(0, T ; \boldsymbol{L}^{2}(\Omega)\right)}^{2}+\|\boldsymbol{H}\|_{H^{2}\left(0, T ; \boldsymbol{L}^{2}(\Omega)\right)}^{2}\right),
\end{aligned}
$$

where the constant $C$ is inherited from Remark 3.5, Corollary 3.6 and Lemma 3.8.

Proof. Taking $\phi=\Delta t \varepsilon_{1}^{k}$ in (3.26a), $\boldsymbol{\psi}=\Delta t \varepsilon_{2}^{k}$ in (3.26b), and $\boldsymbol{\varphi}=\varepsilon_{3}^{k}$ in (3.26c), and following the derivation of (3.15), we obtain

$$
\begin{aligned}
\left(\epsilon_{\infty}+\Delta \epsilon \varpi_{0}^{(\alpha, \beta)}\right)\left\|\varepsilon_{1}^{k}\right\|^{2}+\left\|\varepsilon_{2}^{k}\right\|^{2}= & \epsilon_{\infty}\left(\varepsilon_{1}^{k-1}, \varepsilon_{1}^{k}\right)+\left(\varepsilon_{2}^{k-1}, \varepsilon_{2}^{k}\right)+\Delta \epsilon \sum_{j=1}^{k-1}\left(\varpi_{k-1-j}^{(\alpha, \beta)}-\varpi_{k-j}^{(\alpha, \beta)}\right)\left(\varepsilon_{1}^{j}, \varepsilon_{1}^{k}\right) \\
& +\Delta t\left(\boldsymbol{R}_{1}^{k}+\Delta \epsilon \delta_{t} \boldsymbol{R}_{0}^{k}, \varepsilon_{1}^{k}\right)+\Delta t\left(\boldsymbol{R}_{2}^{k}, \varepsilon_{2}^{k}\right) .
\end{aligned}
$$


Then we can derive from Theorem 3.4 and Remark 3.5 that

$$
\left\|\varepsilon_{1}^{k}\right\|^{2}+\left\|\varepsilon_{2}^{k}\right\|^{2} \leq C \Delta t \sum_{j=1}^{k}\left(\left\|\boldsymbol{R}_{1}^{j}\right\|^{2}+(\Delta \epsilon)^{2}\left\|\delta_{t} \boldsymbol{R}_{0}^{j}\right\|^{2}+\left\|\boldsymbol{R}_{2}^{j}\right\|^{2}\right),
$$

where we used the facts $\epsilon_{\infty} \geq 1, \Delta \epsilon \sum_{j=1}^{k} \varpi_{k-j}^{(\alpha, \beta)}\left\|\varepsilon_{1}^{j}\right\|^{2} \geq 0$, and $\varepsilon_{1}^{0}=\varepsilon_{2}^{0}=\mathbf{0}$.

Similar to the proof of Corollary 3.6, we find from (3.26c) that

$$
\left\|\varepsilon_{3}^{k}\right\| \leq \Delta \epsilon \sum_{j=1}^{k} \varpi_{k-j}^{(\alpha, \beta)}\left\|\varepsilon_{1}^{j}\right\|+\Delta \epsilon\left\|\boldsymbol{R}_{0}^{k}\right\| \leq\left(\Delta \epsilon e_{\alpha, \alpha \beta+1}^{\beta}\left(t_{k} ;-1\right)\right) \max _{1 \leq j \leq k}\left\|\varepsilon_{1}^{j}\right\|+\Delta \epsilon\left\|\boldsymbol{R}_{0}^{k}\right\| .
$$

Finally, applying Lemma 3.8 to (3.31) and (3.32), we arrive at the error estimate (3.30).

Remark 3.10. In principle, we can upgrade the first-order temporal scheme (3.6) to a second-order scheme. The essential component is to apply the piecewise linear approximation to $(2.7 \mathrm{~b})$ that yields

$$
\begin{aligned}
\boldsymbol{P}\left(\boldsymbol{x}, t_{k}\right) & \approx \Delta \epsilon \sum_{j=1}^{k} \int_{t_{j-1}}^{t_{j}} e_{\alpha, \alpha \beta}^{\beta}\left(t_{k}-s ;-1\right)\left\{\frac{t_{j}-s}{t_{j}-t_{j-1}} \boldsymbol{E}^{j-1}(\boldsymbol{x})+\frac{s-t_{j-1}}{t_{j}-t_{j-1}} \boldsymbol{E}^{j}(\boldsymbol{x})\right\} \mathrm{d} s \\
& :=\Delta \epsilon \sum_{j=0}^{k} \rho_{k-j}^{(\alpha, \beta)} \boldsymbol{E}^{j}(\boldsymbol{x}), \quad k \geq 1,
\end{aligned}
$$

where the weights can be computed by

$$
\rho_{k-j}^{(\alpha, \beta)}=\int_{t_{j-1}}^{t_{j}} e_{\alpha, \alpha \beta}^{\beta}\left(t_{k}-s ;-1\right) \frac{s-t_{j-1}}{t_{j}-t_{j-1}} \mathrm{~d} s+\int_{t_{j}}^{t_{j+1}} e_{\alpha, \alpha \beta}^{\beta}\left(t_{k}-s ;-1\right) \frac{t_{j+1}-s}{t_{j+1}-t_{j}} \mathrm{~d} s,
$$

for $1 \leq j \leq k-1$, and $\left\{\rho_{0}^{(\alpha, \beta)}, \rho_{k}^{(\alpha, \beta)}\right\}$ have similar expressions. It is seen that the monotonicity of the weights in Lemma 3.1 has played a critical role in the analysis. However, it is still unknown if $\left\{\rho_{k-j}^{(\alpha, \beta)}\right\}$ enjoys the same property. In fact, we have observed from some numerical evidences that it is parametric dependent as this property is not true for all $\alpha, \beta$.

\section{IMPLEMENTATION AND NUMERICAL RESULTS}

In this section, we follow the idea of $[28,48]$ to introduce a fast temporal convolution algorithm that can alleviate the history dependence of the temporal convolution in the scheme (3.6). It is noteworthy that the semi-discrete scheme and the analysis in Section 3, together with the fast algorithm to be described below, can be incorporated with various spatial discretisation. Compared with the classical Maxwells equations, the most challenging issue in the $\mathrm{H}-\mathrm{N}$ model is the treatment of the temporal convolution with a singular kernel function. Here, we demonstrate the full discretisation via a spectral-Galerkin method for the two-dimensional model. This situation is reminiscent to the comparison of several methods for the Maxwell's equations in [32]: "Of course this is not the setting of real physical interest; however, the two-dimensional case makes a convenient test problem." We remark that one can extend the method to finite element methods or finite differences in two or more dimensions.

\subsection{Fast temporal convolution algorithm}

Among many recent developments of fast algorithms in particular for fractional integral/derivatives, there are a few works on developing fast algorithms for the much more involved kernel function, i.e. the ML function with three parameters. This algorithm can incorporate into (3.6) with different spatial discretisations.

We summarise the algorithm as follows. 
Step 1. Decompose (2.7b) as

$$
\begin{aligned}
\boldsymbol{P}(\boldsymbol{x}, t) & =\Delta \epsilon \int_{t-\Delta t}^{t} e_{\alpha, \alpha \beta}^{\beta}(t-s ;-1) \boldsymbol{E}(\boldsymbol{x}, s) \mathrm{d} s+\Delta \epsilon \int_{0}^{t-\Delta t} e_{\alpha, \alpha \beta}^{\beta}(t-s ;-1) \boldsymbol{E}(\boldsymbol{x}, s) \mathrm{d} s \\
& :=\mathcal{L}(\boldsymbol{E} ; t)+\mathcal{H}(\boldsymbol{E} ; t),
\end{aligned}
$$

where $\mathcal{L}(\boldsymbol{E} ; t)$ and $\mathcal{H}(\boldsymbol{E} ; t)$ are respectively the local and history parts. Corresponding to the discretisation in (3.2), we have

$$
\mathcal{L}\left(\boldsymbol{E} ; t_{k}\right) \approx \mathcal{L}\left(I_{\Delta t} \boldsymbol{E} ; t_{k}\right)=\varpi_{0}^{(\alpha, \beta)} \boldsymbol{E}^{k} ; \quad \mathcal{H}\left(\boldsymbol{E} ; t_{k}\right) \approx \mathcal{H}\left(I_{\Delta t} \boldsymbol{E} ; t_{k}\right) .
$$

We remark that the direct implementation based on the above requires $O\left(N_{t}\right)$ storage and $O\left(N_{t}^{2}\right)$ operations, which is computationally expensive for long time and multi-dimensional simulations. The essence of the fast algorithm is to further approximate the kernel function $e_{\alpha, \alpha \beta}^{\beta}(\cdot ;-1)$ that allows for computing the history part in a recursive manner.

Step 2. Given an integer $B \geq 2$, let $L$ be the smallest integer satisfying $t_{k}<2 B^{L} \Delta t$. For $\ell=1, \cdots, L-1$, we can determine the integer $q_{\ell}$ and $s_{\ell}=q_{\ell} B^{\ell} \Delta t$ such that

$$
t_{k}-s_{\ell} \in I_{\ell}:=\left[B^{\ell-1} \Delta t,\left(2 B^{\ell}-1\right) \Delta t\right] .
$$

As such, we have (see [28])

$$
t_{k}-\Delta t=s_{0}>s_{1}>\cdots>s_{L-1}>s_{L}=0 .
$$

Step 3. Seek the approximation of $e_{\alpha, \alpha \beta}^{\beta}(t ;-1)$ on $I_{\ell}$ by applying the trapezoidal rule to a parametrisation of the contour integral for the inverse Laplace transform:

$$
\begin{aligned}
e_{\alpha, \alpha \beta}^{\beta}(t ;-1) & =\frac{1}{2 \pi i} \int_{\Gamma_{\ell}} \mathscr{L}\left[e_{\alpha, \alpha \beta}^{\beta}(t ;-1)\right](\lambda) e^{t \lambda} \mathrm{d} \lambda=\frac{1}{2 \pi i} \int_{\Gamma_{\ell}} \frac{e^{t \lambda}}{\left(\lambda^{\alpha}+1\right)^{\beta}} \mathrm{d} \lambda \\
& \approx \sum_{j=-N_{\mathrm{col}}}^{N_{\mathrm{col}}-1} \frac{\hat{\omega}_{j}^{(\ell)} e^{t \lambda_{j}^{(\ell)}}}{\left(\left(\lambda_{j}^{(\ell)}\right)^{\alpha}+1\right)^{\beta}},
\end{aligned}
$$

with a precision $\varepsilon_{\mathrm{f}}>0$ and a complex contour $\Gamma_{\ell}$ which can be suitably chosen following the ideas in $[14,28]$. Here, $\hat{\omega}_{j}^{(\ell)}, \lambda_{j}^{(\ell)}$ are the weights and quadrature points for the contour $\Gamma_{\ell}$. The number of quadrature points on $\Gamma_{\ell}, 2 N_{\text {col }}$ is chosen independent of $\ell$.

Step 4. Using (4.2), the history part $\mathcal{H}\left(I_{\Delta t} \boldsymbol{E} ; t_{k}\right)$ can be approximated by

$$
\mathcal{H}\left(I_{\Delta t} \boldsymbol{E} ; t_{k}\right)=\operatorname{Im}\left\{\sum_{\ell=1}^{L} \sum_{j=-N_{\mathrm{col}}}^{N_{\mathrm{col}}-1} \frac{\hat{\omega}_{j}^{(\ell)} e^{\left(t_{k}-s_{\ell-1}\right) \lambda_{j}^{(\ell)}}}{\left[\left(\lambda_{j}^{(\ell)}\right)^{\alpha}+1\right]^{\beta}} \boldsymbol{y}\left(s_{\ell-1}, s_{\ell}, \lambda_{j}^{(\ell)}\right)\right\},
$$

where $\operatorname{Im}\{u\}$ stands for the imaginary part of $u$, and

$$
\boldsymbol{y}(s)=\boldsymbol{y}\left(s, s_{\ell}, \lambda_{j}^{(\ell)}\right)=\int_{s_{\ell}}^{s} e^{-\left(s-s_{\ell}\right) \lambda_{j}^{(\ell)}} I_{\Delta t} \boldsymbol{E}(s) \mathrm{d} s
$$

satisfies the following ODE

$$
\boldsymbol{y}^{\prime}(s)=\lambda_{j}^{(\ell)} \boldsymbol{y}(s)+I_{\Delta t} \boldsymbol{E}(s), \quad \boldsymbol{y}\left(s_{\ell}\right)=0 .
$$


Remark 4.1. This fast convolution algorithm has the same storage and computational cost as that in [28], i.e. it requires $O\left(\log N_{t}\right)$ storage and $O\left(N_{t} \log N_{t}\right)$ operations over $N_{t}$ time steps, when only cost in time direction is considered. However, the direct implementation of the scheme (3.2) would require $O\left(N_{t}\right)$ storage and $O\left(N_{t}^{2}\right)$ operations, which is computational expensive and forms a bottleneck for long time simulation. It is worthy noting that the kernel function in H-N model (see (2.8)) is much more complex than the kernel functions in $[28,48]$ and references therein, so how to develop a fast convolution algorithm for the H-N model is much more involved. We also point out that some different approaches were developed in $[5,47]$.

\subsection{Full discretisation of a two-dimensional H-N model}

As an illustration of full-discrete scheme, we consider the spatial discretisation of the H-N model using the spectral-Galerkin method in two dimensions. More precisely, we consider (2.7) on the rectangular domain $\Omega=(a, b) \times(c, d)$ of the form:

$$
\begin{array}{ll}
\epsilon_{\infty} \partial_{t} \boldsymbol{E}+\partial_{t} \boldsymbol{P}=\operatorname{curl} H, \quad \partial_{t} H=-\operatorname{curl} \boldsymbol{E} & \text { in } \Omega \times(0, T], \\
\boldsymbol{P}(x, y, t)=\Delta \epsilon \int_{0}^{t} e_{\alpha, \alpha \beta}^{\beta}(t-s ;-1) \boldsymbol{E}(x, y, s) \mathrm{d} s & \text { in } \Omega \times(0, T], \\
\boldsymbol{E}(x, y, 0)=\boldsymbol{E}_{0}(x, y), \quad H(x, y, 0)=H_{0}(x, y), & \text { in } \Omega, \\
E_{x}(x, c, t)=E_{x}(x, d, t)=E_{y}(a, y, t)=E_{y}(b, y, t)=0 & \text { for } t \in(0, T],
\end{array}
$$

where $\boldsymbol{E}=\left(E_{x}, E_{y}\right)^{T}$ and $\boldsymbol{P}=\left(P_{x}, P_{y}\right)^{T}$ are vectors, but $H$ is a scalar unknown. Recap on the two-types of curl operators:

$$
\operatorname{curl} H=\left(\partial_{y} H,-\partial_{x} H\right)^{T}, \quad \operatorname{curl} \boldsymbol{E}=\partial_{y} E_{x}-\partial_{x} E_{y} .
$$

Let $\mathbb{P}_{N}$ be the space of the algebraic polynomials in one variable of degree not more than $N$, and let $\mathbb{P}_{N}^{0}$ be the subspace of $\mathbb{P}_{N}$, where each polynomial vanishes at the two end-points of the interval. We further denote $V_{N}=\mathbb{P}_{N} \times \mathbb{P}_{N}$, and define

$$
\boldsymbol{V}_{N}^{0}=\left\{(u, v)^{T} \in\left(V_{N}\right)^{2}:\left.u\right|_{y=c}=\left.u\right|_{y=d}=0,\left.v\right|_{x=a}=\left.v\right|_{x=b}=0\right\} .
$$

The full-discrete scheme for (2.1) is to find $\boldsymbol{E}_{N}^{k}, \boldsymbol{P}_{N}^{k} \in \boldsymbol{V}_{N}^{0}$ and $H_{N}^{k} \in V_{N}$ such that for $k \geq 1$,

$$
\begin{array}{ll}
\epsilon_{\infty}\left(\delta_{t} \boldsymbol{E}_{N}^{k}, \boldsymbol{\phi}\right)+\left(\delta_{t} \boldsymbol{P}_{N}^{k}, \boldsymbol{\phi}\right)=\left(\operatorname{curl} H_{N}^{k}, \boldsymbol{\phi}\right), & \forall \boldsymbol{\phi} \in \boldsymbol{V}_{N}^{0}, \\
\left(\delta_{t} H_{N}^{k}, \psi\right)=-\left(\operatorname{curl} \boldsymbol{E}_{N}^{k}, \psi\right), & \forall \psi \in V_{N}, \\
\left(\boldsymbol{P}_{N}^{k}, \boldsymbol{\varphi}\right)=\Delta \epsilon \sum_{j=1}^{k} \varpi_{k-j}^{(\alpha, \beta)}\left(\boldsymbol{E}_{N}^{j}, \boldsymbol{\varphi}\right), & \forall \boldsymbol{\varphi} \in \boldsymbol{V}_{N}^{0},
\end{array}
$$

where the initial values are

$$
\boldsymbol{E}_{N}^{0}=\mathcal{I}_{N} \boldsymbol{E}_{0}, \quad H_{N}^{0}=\mathcal{I}_{N} H_{0}, \quad \boldsymbol{P}_{N}^{0}=\mathbf{0} .
$$

Here, $\mathcal{I}_{N}: C(\bar{\Omega}) \rightarrow V_{N}$ is the tensorial Legendre-Gauss-Lobatto (LGL) interpolation operator.

Remark 4.2. Note that $\boldsymbol{E}_{N}^{k}, \boldsymbol{P}_{N}^{k}$ and $H_{N}^{k}$ are expansions in terms of Legendre basis polynomials. Then taking the divergence of (4.4a), we find that $\nabla \cdot\left(\epsilon_{\infty} \boldsymbol{E}_{N}^{k}+\boldsymbol{P}_{N}^{k}\right)=0$ as we can show that $\nabla \cdot\left(\right.$ curl $\left.H_{N}^{k}\right) \equiv 0$. Indeed, we can write $H_{N}^{k}$ in terms of the Legendre polynomials:

$$
H_{N}^{k}=\sum_{i=0}^{N} \sum_{j=0}^{N} \tilde{H}_{i j}^{k} L_{i}(x) L_{j}(y),
$$


where $\tilde{H}_{i j}^{k}$ are the expansion coefficients. From direct calculation, we obtain

$$
\nabla \cdot\left(\operatorname{curl} H_{N}^{k}\right)=\sum_{i=0}^{N} \sum_{j=0}^{N} \tilde{H}_{i j}^{k}\left(L_{i}^{\prime}(x) L_{j}^{\prime}(y)-L_{i}^{\prime}(x) L_{j}^{\prime}(y)\right) \equiv 0 .
$$

Therefore, we can claim $\nabla \cdot \boldsymbol{P}_{N}^{k}=0$ and $\nabla \cdot \boldsymbol{E}_{N}^{k}=0$ like in the derivation in Remark 3.3. However, we note that the discrete magnetic field $H_{N}^{k}$ is a scalar, thus the divergence of it is not defined [32]. We shall provide some numerical verifications in Table 2.

The stability and well-posedness of the scheme (4.4) is a direct consequence of Theorem 3.4.

Theorem 4.3. The full-discrete scheme (4.4) is unconditionally stable in the sense that for all $\Delta t>0$,

$$
\mathscr{E}_{N}^{k} \leq \mathscr{E}_{N}^{k-1} \leq \cdots \leq \mathscr{E}_{N}^{0}, \quad k \geq 1
$$

where $\mathscr{E}_{N}^{0}:=\epsilon_{\infty}\left\|\boldsymbol{E}_{N}^{0}\right\|^{2}+\left\|H_{N}^{0}\right\|^{2}$ and

$$
\mathscr{E}_{N}^{k}:=\epsilon_{\infty}\left\|\boldsymbol{E}_{N}^{k}\right\|^{2}+\left\|H_{N}^{k}\right\|^{2}+\Delta \epsilon \sum_{j=1}^{k} \varpi_{k-j}^{(\alpha, \beta)}\left\|\boldsymbol{E}_{N}^{j}\right\|^{2}, \quad k \geq 1 .
$$

Following the argument for proving Theorem 3.9, we can show the convergence. To this end, we sketch the proof with an emphasis on the estimation of spatial error.

Let $\boldsymbol{E}_{*}=\left(E_{x *}, E_{y *}\right)^{T} \in \boldsymbol{V}_{N}^{0}, \boldsymbol{P}_{*}=\left(P_{x *}, P_{y *}\right)^{T} \in \boldsymbol{V}_{N}^{0}$, and $H_{*} \in V_{N}$ be some suitable orthogonal projections to be specified later. We introduce

$$
\begin{array}{lll}
\boldsymbol{e}_{1}^{k}=\boldsymbol{E}_{N}^{k}-\boldsymbol{E}_{*}^{k}, & e_{2}^{k}=H_{N}^{k}-H_{*}^{k}, & \boldsymbol{e}_{3}^{k}=\boldsymbol{P}_{N}^{k}-\boldsymbol{P}_{*}^{k}, \\
\boldsymbol{\eta}_{1}^{k}=\left.\boldsymbol{E}\right|_{t_{k}}-\boldsymbol{E}_{*}^{k}, & \eta_{2}^{k}=\left.H\right|_{t_{k}}-H_{*}^{k}, & \boldsymbol{\eta}_{3}^{k}=\left.\boldsymbol{P}\right|_{t_{k}}-\boldsymbol{P}_{*}^{k} .
\end{array}
$$

We infer from (2.1) to (4.4) the error equations:

$$
\begin{aligned}
& \epsilon_{\infty}\left(\delta_{t} \boldsymbol{e}_{1}^{k}, \boldsymbol{\phi}\right)+\left(\delta_{t} \boldsymbol{e}_{3}^{k}, \boldsymbol{\phi}\right)-\left(e_{2}^{k}, \operatorname{curl} \boldsymbol{\phi}\right)=\left(\delta_{t} \boldsymbol{\eta}_{3}^{k}, \boldsymbol{\phi}\right)+\left(\boldsymbol{f}_{1}^{k}, \boldsymbol{\phi}\right), \\
& \left(\delta_{t} e_{2}^{k}, \psi\right)+\left(\operatorname{curl} \boldsymbol{e}_{1}^{k}, \psi\right)=\left(f_{2}^{k}, \psi\right), \\
& \left(\boldsymbol{e}_{3}^{k}, \boldsymbol{\varphi}\right)=\Delta \epsilon \sum_{j=1}^{k} \varpi_{k-j}^{(\alpha, \beta)}\left(\boldsymbol{e}_{1}^{j}, \boldsymbol{\varphi}\right)+\left(\boldsymbol{\eta}_{3}^{k}, \boldsymbol{\varphi}\right)-\Delta \epsilon\left(\boldsymbol{f}_{3}^{k}, \boldsymbol{\varphi}\right),
\end{aligned}
$$

where

$$
\boldsymbol{f}_{1}^{k}:=\boldsymbol{R}_{1}^{k}+\epsilon_{\infty} \delta_{t} \boldsymbol{\eta}_{1}^{k}-\operatorname{curl} \eta_{2}^{k}, \quad f_{2}^{k}:=R_{2}^{k}+\delta_{t} \eta_{2}^{k}+\operatorname{curl} \boldsymbol{\eta}_{1}^{k}, \quad \boldsymbol{f}_{3}^{k}:=\sum_{j=1}^{k} \varpi_{k-j}^{(\alpha, \beta)} \boldsymbol{\eta}_{1}^{j}+\boldsymbol{R}_{0}^{k} .
$$

Here, $\boldsymbol{R}_{0}^{k}, \boldsymbol{R}_{1}^{k}$, and $R_{2}^{k}$ are defined in (3.3) and (3.27) with reduction to the two-dimensional setting. Like (3.31), we can derive

$$
\left\|\boldsymbol{e}_{1}^{k}\right\|^{2}+\left\|e_{2}^{k}\right\|^{2} \leq C\left(\left\|\boldsymbol{e}_{1}^{0}\right\|^{2}+\left\|e_{2}^{0}\right\|^{2}+\Delta t \sum_{j=1}^{k}\left(\left\|\boldsymbol{f}_{1}^{j}\right\|^{2}+(\Delta \epsilon)^{2}\left\|\delta_{t} \boldsymbol{f}_{3}^{j}\right\|^{2}+\left\|f_{2}^{j}\right\|^{2}\right)\right),
$$

and similar to the proof of Corollary 3.6 , we can obtain

$$
\left\|\boldsymbol{e}_{3}^{k}\right\| \leq C \max _{1 \leq j \leq k}\left\|\boldsymbol{e}_{1}^{j}\right\|+\left\|\boldsymbol{\eta}_{3}^{k}\right\|+\Delta \epsilon\left\|\boldsymbol{f}_{3}^{k}\right\|
$$


Recall that Lemma 3.8 provides the error bounds of $\boldsymbol{R}_{0}^{k}, \boldsymbol{R}_{1}^{k}$, and $R_{2}^{k}$, so it suffices to estimate the errors involving $\boldsymbol{\eta}_{1}^{k}, \eta_{2}^{k}$ and $\boldsymbol{\eta}_{3}^{k}$. We first deal with the summation in $\boldsymbol{f}_{3}^{k}$. Following the same lines as deriving the last estimate in Lemma 3.8 , one has

$$
\begin{aligned}
\left\|\delta_{t}\left(\sum_{i=1}^{j} \varpi_{j-i}^{(\alpha, \beta)} \boldsymbol{\eta}_{1}^{i}\right)\right\| & =\frac{1}{\Delta t}\left\|\sum_{i=1}^{j} \varpi_{j-i}^{(\alpha, \beta)} \boldsymbol{\eta}_{1}^{i}-\sum_{i=1}^{j-1} \varpi_{j-1-i}^{(\alpha, \beta)} \boldsymbol{\eta}_{1}^{i}\right\| \\
& \leq \frac{1}{\Delta t}\left\|\sum_{i=1}^{j} \int_{t_{i-1}}^{t_{i}} e_{\alpha, \alpha \beta}^{\beta}\left(t_{j}-s ;-1\right) \boldsymbol{\eta}_{1}^{i} \mathrm{~d} s-\sum_{i=1}^{j-1} \int_{t_{i-1}}^{t_{i}} e_{\alpha, \alpha \beta}^{\beta}\left(t_{j-1}-s ;-1\right) \boldsymbol{\eta}_{1}^{i} \mathrm{~d} s\right\| \\
& =\frac{1}{\Delta t}\left\|\int_{0}^{t_{1}} e_{\alpha, \alpha \beta}^{\beta}\left(t_{j}-s ;-1\right) \boldsymbol{\eta}_{1}^{1} \mathrm{~d} s+\sum_{i=1}^{j-1} \int_{t_{i-1}}^{t_{i}} e_{\alpha, \alpha \beta}^{\beta}\left(t_{j-1}-s ;-1\right)\left(\boldsymbol{\eta}_{1}^{i+1}-\boldsymbol{\eta}_{1}^{i}\right) \mathrm{d} s\right\| \\
& \leq e_{\alpha, \alpha \beta}^{\beta}\left(t_{j-1} ;-1\right)\left\|\boldsymbol{\eta}_{1}^{1}\right\|+\sum_{i=1}^{j-1} \int_{t_{i-1}}^{t_{i}} e_{\alpha, \alpha \beta}^{\beta}\left(t_{j-1}-s ;-1\right)\left\|\delta_{t} \boldsymbol{\eta}_{1}^{i+1}\right\| \mathrm{d} s .
\end{aligned}
$$

We proceed with introducing some orthogonal projections, and review the relevant approximation results in [44]. Let $\pi_{N, x}^{1}: H^{1}\left(I_{x}\right) \rightarrow \mathbb{P}_{N}$ be the $H^{1}$-orthogonal projection, and let $\pi_{N, x}^{1,0}: H_{0}^{1}\left(I_{x}\right) \rightarrow \mathbb{P}_{N}^{0}$ be the $H_{0}^{1}$ orthogonal projection. Likewise, we can define the operators $\pi_{N, y}^{1}$ and $\pi_{N, y}^{1,0}$ on the interval $I_{y}$. Here we choose

$$
\left\{E_{x *} ; P_{x *}\right\}=\left(\pi_{N, x}^{1} \circ \pi_{N, y}^{1,0}\right)\left\{E_{x} ; P_{x}\right\}, \quad\left\{E_{y *} ; P_{y *}\right\}=\left(\pi_{N, x}^{1,0} \circ \pi_{N, x}^{1}\right)\left\{E_{y} ; P_{y}\right\}, \quad H_{*}=\left(\pi_{N, x}^{1} \circ \pi_{N, y}^{1}\right) H .
$$

According to [44], we have

$$
\left\|U_{x *}-U_{x}\right\|_{H^{s}(\Omega)} \leq c N^{s-r}\left\|U_{x}\right\|_{H^{r}(\Omega)},\left\|U_{y *}-U_{y}\right\|_{H^{s}(\Omega)} \leq c N^{s-r}\left\|U_{y}\right\|_{H^{r}(\Omega)}, \quad s=0,1, \quad r \geq 1,
$$

and

$$
\left\|U-\mathcal{I}_{N} U\right\| \leq c N^{-r}\|U\|_{H^{r}(\Omega)}, \quad r \geq 1 .
$$

Below, we shall set $U$ to be the unknowns. Now we are in a position to give the error estimates involving $\boldsymbol{\eta}_{1}^{k}, \eta_{2}^{k}$ and $\boldsymbol{\eta}_{3}^{k}$. From (4.9), we have

$$
\begin{aligned}
\left\|\boldsymbol{\eta}_{1}^{j}\right\| & \leq c N^{-r}\left(\left\|E_{x}\left(\cdot, t_{j}\right)\right\|_{H^{r}(\Omega)}+\left\|E_{y}\left(\cdot, t_{j}\right)\right\|_{H^{r}(\Omega)}\right) \leq c N^{-r}\|\boldsymbol{E}\|_{L^{\infty}\left(0, T ; \boldsymbol{H}^{r}(\Omega)\right)} \\
\left\|\boldsymbol{\eta}_{3}^{k}\right\| & \leq c N^{-r}\left(\left\|P_{x}\left(\cdot, t_{k}\right)\right\|_{H^{r}(\Omega)}+\left\|P_{y}\left(\cdot, t_{k}\right)\right\|_{H^{r}(\Omega)}\right) \leq c N^{-r}\|\boldsymbol{P}\|_{L^{\infty}\left(0, T ; \boldsymbol{H}^{r}(\Omega)\right)}, \\
\left\|\delta_{t} \boldsymbol{\eta}_{1}^{k}\right\| & \leq c\left\|\partial_{t} \boldsymbol{\eta}_{1}^{k}\right\| \leq c N^{-r}\left(\left\|\partial_{t} E_{x}\left(\cdot, t_{k}\right)\right\|_{H^{r}(\Omega)}+\left\|\partial_{t} E_{y}\left(\cdot, t_{k}\right)\right\|_{H^{r}(\Omega)}\right) \leq c N^{-r}\left\|\partial_{t} \boldsymbol{E}\right\|_{L^{\infty}\left(0, T ; \boldsymbol{H}^{r}(\Omega)\right)}, \\
\left\|\operatorname{curl} \boldsymbol{\eta}_{1}^{k}\right\| & =\left\|\partial_{y}\left(E_{x}-E_{x *}\right)-\partial_{x}\left(E_{y}-E_{y *}\right)\right\| \leq\left\|\partial_{y}\left(E_{x}-E_{x *}\right)\right\|+\left\|\partial_{x}\left(E_{y}-E_{y *}\right)\right\| \\
& \leq c N^{1-r}\left(\left\|E_{x}\left(\cdot, t_{k}\right)\right\|_{H^{r}(\Omega)}+\left\|E_{y}\left(\cdot, t_{k}\right)\right\|_{H^{r}(\Omega)}\right) \leq c N^{(1-r)}\|\boldsymbol{E}\|_{L^{\infty}\left(0, T ; \boldsymbol{H}^{r}(\Omega)\right)}, \\
\left\|\delta_{t} \eta_{2}^{k}\right\| & \leq c\left\|\partial_{t} \eta_{2}^{k}\right\| \leq c N^{-r}\left\|\partial_{t} H\left(\cdot, t_{k}\right)\right\|_{H^{r}(\Omega)} \leq c N^{-r}\left\|\partial_{t} H\right\|_{L^{\infty}\left(0, T ; H^{r}(\Omega)\right)},
\end{aligned}
$$

and

$$
\left\|\operatorname{curl} \eta_{2}^{k}\right\|=\left\|\partial_{x} \eta_{2}^{k}\right\|+\left\|\partial_{y} \eta_{2}^{k}\right\| \leq c N^{(1-r)}\left\|H^{k}\right\|_{H^{r}(\Omega)} \leq c N^{(1-r)}\|H\|_{L^{\infty}\left(0, T ; H^{r}(\Omega)\right)} .
$$

Using the triangular inequality and the approximation results (4.9) and (4.10), we obtain

$$
\left\|\boldsymbol{e}_{1}^{0}\right\| \leq c N^{-r}\left\|\boldsymbol{E}_{0}\right\|_{\boldsymbol{H}^{r}(\Omega)}, \quad\left\|e_{2}^{0}\right\| \leq c N^{-r}\left\|H_{0}\right\|_{H^{r}(\Omega)},
$$

for the initial errors. Collecting all the estimates above, and noting (4.8), we present the following convergence result. 
TABLE 1. Errors and convergence rates of the fast temporal convolution algorithm.

\begin{tabular}{llllllllll}
\hline \hline$\Delta t$ & \multirow{2}{*}{ ErrE $_{\mathrm{F}}$} & Order & ErrH $_{\mathrm{F}}$ & Order & ErrP $_{\mathrm{F}}$ & Order & ErrE $_{\mathrm{DF}}$ & ErrH $_{\mathrm{DF}}$ & ErrP $_{\mathrm{DF}}$ \\
\hline $2^{-4}$ & $6.4914 \mathrm{e}-03$ & - & $2.0905 \mathrm{e}-03$ & - & $6.1861 \mathrm{e}-03$ & - & $2.8917 \mathrm{e}-16$ & $1.4197 \mathrm{e}-16$ & $2.5075 \mathrm{e}-16$ \\
$2^{-6}$ & $1.6836 \mathrm{e}-03$ & 0.99 & $4.0510 \mathrm{e}-04$ & 1.17 & $1.7062 \mathrm{e}-03$ & 0.94 & $3.6776 \mathrm{e}-16$ & $1.7787 \mathrm{e}-16$ & $1.4391 \mathrm{e}-16$ \\
$2^{-8}$ & $4.1803 \mathrm{e}-04$ & 1.01 & $8.3436 \mathrm{e}-05$ & 1.13 & $4.5249 \mathrm{e}-04$ & 0.96 & $2.7708 \mathrm{e}-16$ & $4.3866 \mathrm{e}-16$ & $5.4712 \mathrm{e}-17$ \\
$2^{-10}$ & $1.0307 \mathrm{e}-04$ & 1.01 & $1.7985 \mathrm{e}-05$ & 1.10 & $1.1771 \mathrm{e}-04$ & 0.97 & $3.8889 \mathrm{e}-16$ & $5.4694 \mathrm{e}-16$ & $5.9642 \mathrm{e}-17$ \\
$2^{-12}$ & $2.5458 \mathrm{e}-05$ & 1.01 & $4.0045 \mathrm{e}-06$ & 1.08 & $3.0253 \mathrm{e}-05$ & 0.98 & $1.6182 \mathrm{e}-15$ & $1.8799 \mathrm{e}-16$ & $1.3962 \mathrm{e}-15$ \\
$2^{-14}$ & $6.3060 \mathrm{e}-06$ & 1.01 & $9.1495 \mathrm{e}-07$ & 1.06 & $7.7104 \mathrm{e}-06$ & 0.99 & $4.6538 \mathrm{e}-16$ & $3.1352 \mathrm{e}-15$ & $1.2561 \mathrm{e}-15$ \\
\hline
\end{tabular}

Theorem 4.4. Let $\boldsymbol{E}_{N}^{k}, \boldsymbol{P}_{N}^{k}, H_{N}^{k}$ be the solution of (4.4) that approximates the solution of (2.1). Assume

$$
E_{x}, P_{x} \in H^{2}\left(0, T ; H^{r}(\Omega) \cap\left(H^{1}\left(I_{x}\right) \otimes H_{0}^{1}\left(I_{y}\right)\right)\right), E_{y}, P_{y} \in H^{2}\left(0, T ; H^{r}(\Omega) \cap\left(H_{0}^{1}\left(I_{x}\right) \otimes H^{1}\left(I_{y}\right)\right)\right),
$$

and $H \in H^{2}\left(0, T ; H^{r}(\Omega)\right)$, then for $k \geq 1$,

$$
\begin{aligned}
\| \boldsymbol{E}\left(\cdot, t_{k}\right) & -\boldsymbol{E}_{N}^{k}\left\|^{2}+\right\| H\left(\cdot, t_{k}\right)-H_{N}^{k}\left\|^{2}+\right\| \boldsymbol{P}\left(\cdot, t_{k}\right)-\boldsymbol{P}_{N}^{k} \|^{2} \\
\leq & C(\Delta t)^{2}\left(\|\boldsymbol{E}\|_{H^{2}\left(0, T ; \boldsymbol{L}^{2}(\Omega)\right)}^{2}+\|\boldsymbol{P}\|_{H^{2}\left(0, T ; \boldsymbol{L}^{2}(\Omega)\right)}^{2}+\|H\|_{H^{2}\left(0, T ; L^{2}(\Omega)\right)}^{2}\right) \\
& +C N^{2(1-r)}\left(N^{-2}\left\|\boldsymbol{E}_{0}\right\|_{\boldsymbol{H}^{r}(\Omega)}^{2}+N^{-2}\left\|H_{0}\right\|_{H^{r}(\Omega)}^{2}+N^{-2}\left\|\partial_{t} \boldsymbol{E}\right\|_{L^{\infty}\left(0, T, \boldsymbol{H}^{r}(\Omega)\right)}^{2}+N^{-2}\left\|\partial_{t} H\right\|_{L^{\infty}\left(0, T ; H^{r}(\Omega)\right)}^{2}\right. \\
& \left.+N^{-2}\|\boldsymbol{E}\|_{L^{\infty}\left(0, T, \boldsymbol{H}^{r}(\Omega)\right)}^{2}+N^{-2}\|\boldsymbol{P}\|_{L^{\infty}\left(0, T ; \boldsymbol{H}^{r}(\Omega)\right)}^{2}+\|H\|_{L^{\infty}\left(0, T ; H^{r}(\Omega)\right)}^{2}+\|\boldsymbol{E}\|_{L^{\infty}\left(0, T ; \boldsymbol{H}^{r}(\Omega)\right)}^{2}\right)
\end{aligned}
$$

for a suitable $\Delta t$ (see Thm. 3.4 and Rem. 3.5). Here, $C$ is a positive constant independent of $\Delta t, N$ and any function.

\subsection{Numerical results}

In this subsection, we provide ample numerical results to show the efficiency and accuracy of the proposed methods with a focus on the performance of the treatment in time discretisation.

\subsubsection{Accuracy and efficiency tests}

Consider the system (2.1) with the exact solution:

$$
\begin{aligned}
& \boldsymbol{E}(x, y, t)=\frac{t^{4}}{\Gamma(5)} \boldsymbol{w}(x, y), \quad \boldsymbol{P}(x, y, t)=\Delta \epsilon e_{\alpha, \alpha \beta+5}^{\beta}(t ;-1) \boldsymbol{w}(x, y), \boldsymbol{w}(x, y)=\left(\begin{array}{r}
-\cos (\pi x) \sin (\pi y) \\
\sin (\pi x) \cos (\pi y)
\end{array}\right), \\
& H(x, y, t)=\left(\frac{4 \epsilon_{\infty}}{\pi \Gamma(5)} t^{3}+\Delta \epsilon e_{\alpha, \alpha \beta+4}^{\beta}(t ;-1)\right) \cos (\pi x) \cos (\pi y) .
\end{aligned}
$$

As such, the second equation in (2.1) must have a source term

$$
f(x, y, t)=\left(\frac{2 \pi}{\Gamma(5)} t^{4}+\frac{12 \epsilon_{\infty}}{\pi \Gamma(5)} t^{2}+\frac{\Delta \epsilon}{\pi} e_{\alpha, \alpha \beta+3}^{\beta}(t ;-1)\right) \cos (\pi x) \cos (\pi y),
$$

which one can verify by using the formulas in [24], (2.10) and (2.26).

For notational simplicity, we denote by $U_{N, D}^{k}$ and $U_{N, F}^{k}$ the numerical solutions derived by the direct and fast algorithms at $t_{k}=k \Delta t$. Correspondingly, we denote the discrete $L^{2}$-errors by $\operatorname{Err}_{\mathrm{F}}:=\left\|U\left(\cdot, t_{k}\right)-U_{N, F}^{k}\right\|_{N}$ and $\operatorname{ErrU}_{\mathrm{DF}}:=\left\|U_{N, D}^{k}-U_{N, F}^{k}\right\|_{N}$, respectively, where $U$ can be $\boldsymbol{E}, H$ or $\boldsymbol{P}$. In the following tests, we take $\Omega=(-1,1)^{2}$ and $\epsilon_{\infty}=\Delta \epsilon=1$.

Firstly, in Table 1, we tabulate the discrete $L^{2}$-errors between the exact and numerical solutions, together with convergence orders, obtained by the schemes with $\alpha=\beta=0.5$ and $N=50$ at $T=1$. In the rightmost 


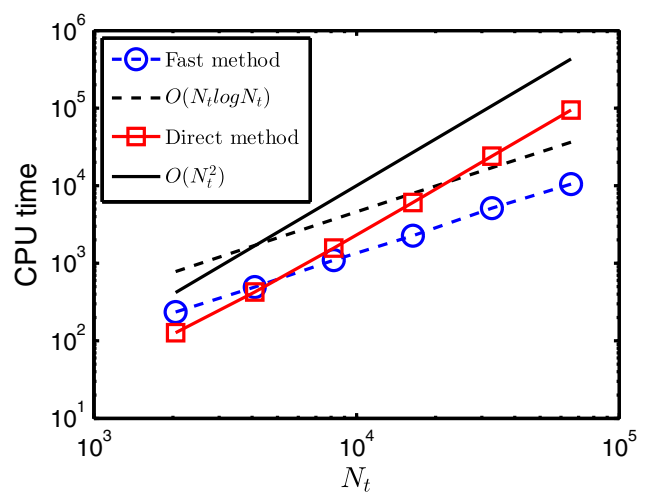

FiguRE 1. Direct versus fast algorithms in $\log -\log$ scale.

three columns, we list the errors between the numerical solutions by direct and fast algorithms (with $N_{\text {col }}=30$ ), which are apparently negligible. We also observe that the first-order convergence as expected.

Secondly, we compare in Figure 1 the computational time in seconds against $N_{t}$ between the direct and fast convolution algorithms with $\alpha=\beta=0.5, N=50, N_{\text {col }}=30$ and with different $\Delta t$ at $T=1$. Note that the fast convolution algorithm requires $O\left(N_{t} \log N_{t}\right)$ operations over $N_{t}$ time steps, while the direct algorithm requires $O\left(N_{t}^{2}\right)$ operations. As such, much saving can be achieved by using the fast convolution algorithm which is therefore necessary for long time simulation.

Thirdly, we depict in Figures 2 and 3 the convergence rates in both time and space with different parameters $\alpha, \beta$. As expected, we observe from Figure 2 the first-order convergence order in time, while from Figure 3 the spectral accuracy in space (given the spatial smooth exact solution). Here, we understand $O(\Delta t)=0.5 \Delta t$. For the latter, we choose $\Delta t=0.00001$ so that we can demonstrate the spatial errors. Indeed, the numerics confirm the convergence $O\left(\Delta t+e^{-c N}\right)$ for some $c>0$.

Finally, we tabulate the discrete $L^{\infty}$-norm of the divergence of electric and polarisation fields with $\Delta t=0.001$, $N=50$ and different $\alpha, \beta$ at different times $t_{k}=k \Delta t$ in Table 2, which shows the scheme can preserve this property well.

\subsubsection{Discrete energy decay}

In order to illustrate the discrete energy dissipation shown in Theorem 4.3, we set the initial values to be

$$
E_{x}(x, y, 0)=\frac{1}{\sqrt{2}} \cos (\pi x) \sin (\pi y), \quad E_{y}(x, y, 0)=-\frac{1}{\sqrt{2}} \sin (\pi x) \cos (\pi y), \quad H(x, y, 0)=0 .
$$

Note that the system must be homogeneous to possess such a property (see (4.4)). As a result, we use sufficiently fine mesh grids to verify the accuracy and convergence order as observed previously. Here, we record in Figure 4 the evolution of the discrete energy $\mathscr{E}_{N}^{k}$ obtained by the scheme with $\Delta t=0.01, N=50$ and $N_{\text {col }}=30$ for some different parameters $\alpha, \beta$. Indeed, these numerical evidences validate this behaviour. Interestingly, when it comes to the discrete analogue of the energy in Theorem 2.5: $\tilde{\mathscr{E}}_{N}^{k}:=\epsilon_{\infty}\left\|\boldsymbol{E}_{N}^{k}\right\|^{2}+\left\|H_{N}^{k}\right\|^{2}$, we observe from Figure 5 that it fails to satisfy this decaying property. Indeed, as shown in Theorem 2.5, this energy at continuous level can only be controlled by the initial energy. In fact, a similar behaviour has been observed for the Cole-Cole model in [21]. 


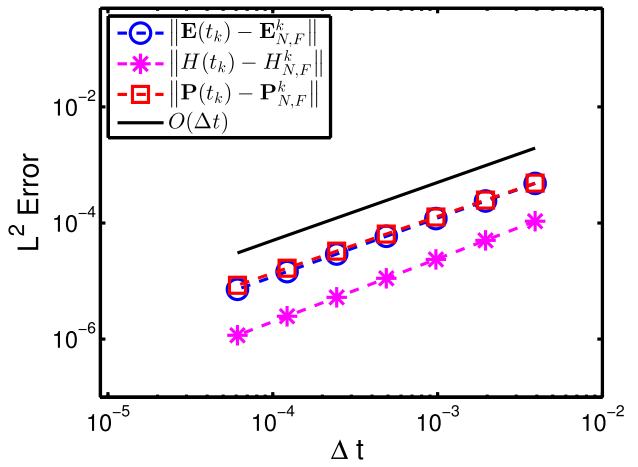

(a)

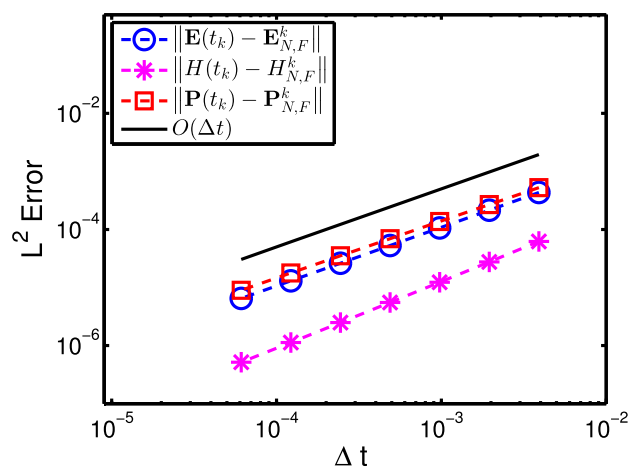

(c)

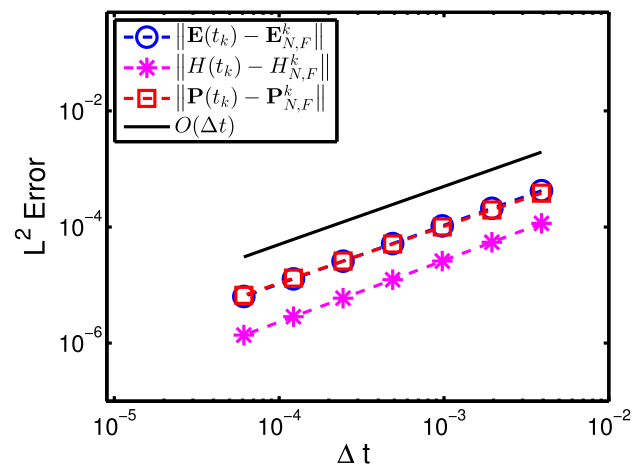

(b)

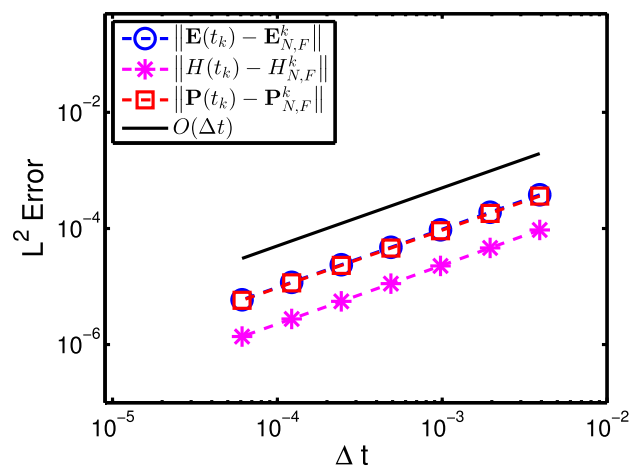

(d)

Figure 2. Convergence order in time with $N=50, N_{\text {col }}=30$ and different parameters $\alpha, \beta$ in $\log -\log$ scale. (a) $\alpha=0.3, \beta=0.3$. (b) $\alpha=0.3, \beta=0.7$. (c) $\alpha=0.7, \beta=0.3$. (d) $\alpha=0.7, \beta=0.7$.

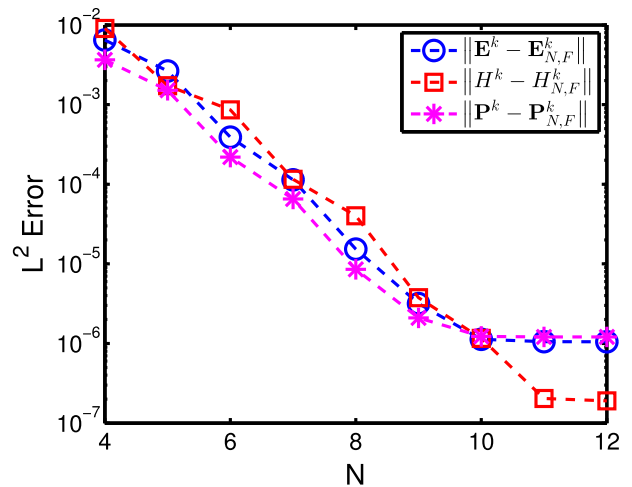

(a)

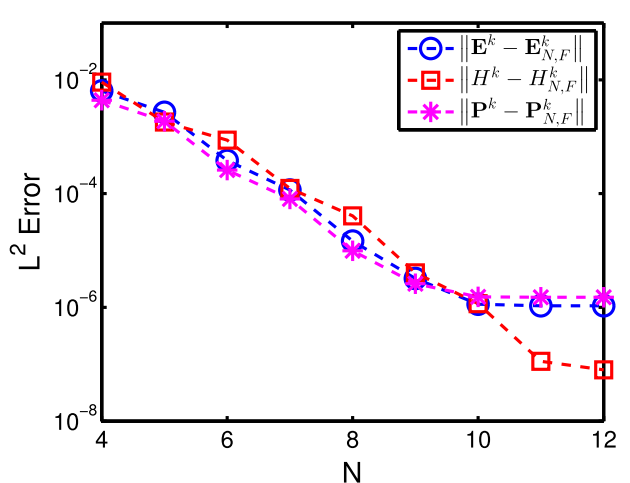

(b)

Figure 3. Convergence behaviour in space for different parameters $\alpha, \beta$ in semi-log scale. (a) $\alpha=0.3, \beta=0.6$. (b) $\alpha=0.6, \beta=0.3$. 
TABLE 2. Discrete $L^{\infty}$-norm of the divergence of electric and polarisation fields.

\begin{tabular}{lccccccc}
\hline \hline \multirow{2}{*}{$k$} & \multicolumn{2}{c}{$\alpha=0.3, \quad \beta=0.7$} & $\alpha=0.5$, & $\beta=0.5$ & $\alpha=0.7, \quad \beta=0.3$ \\
\cline { 2 - 7 } & $\left\|\nabla \cdot \boldsymbol{E}^{k}\right\|_{\infty}$ & $\left\|\nabla \cdot \boldsymbol{P}^{k}\right\|_{\infty}$ & $\left\|\nabla \cdot \boldsymbol{E}^{k}\right\|_{\infty}$ & $\left\|\nabla \cdot \boldsymbol{P}^{k}\right\|_{\infty}$ & $\left\|\nabla \cdot \boldsymbol{E}^{k}\right\|_{\infty}$ & $\left\|\nabla \cdot \boldsymbol{P}^{k}\right\|_{\infty}$ \\
\hline \multirow{2}{*}{200} & $1.3034 \mathrm{e}-16$ & $5.3398 \mathrm{e}-17$ & $1.3084 \mathrm{e}-16$ & $5.3862 \mathrm{e}-17$ & $1.2869 \mathrm{e}-16$ & $6.4280 \mathrm{e}-17$ \\
400 & $2.2010 \mathrm{e}-15$ & $1.0001 \mathrm{e}-15$ & $2.2415 \mathrm{e}-15$ & $1.0719 \mathrm{e}-15$ & $2.2190 \mathrm{e}-15$ & $1.2638 \mathrm{e}-15$ \\
600 & $1.1832 \mathrm{e}-14$ & $5.6432 \mathrm{e}-15$ & $1.1797 \mathrm{e}-14$ & $6.1230 \mathrm{e}-15$ & $1.1630 \mathrm{e}-14$ & $7.1018 \mathrm{e}-15$ \\
800 & $3.9361 \mathrm{e}-14$ & $1.9593 \mathrm{e}-14$ & $3.9541 \mathrm{e}-14$ & $2.1420 \mathrm{e}-14$ & $3.8714 \mathrm{e}-14$ & $2.4878 \mathrm{e}-14$ \\
1000 & $1.0110 \mathrm{e}-13$ & $5.1943 \mathrm{e}-14$ & $1.0217 \mathrm{e}-13$ & $5.7495 \mathrm{e}-14$ & $9.9812 \mathrm{e}-14$ & $6.5602 \mathrm{e}-14$ \\
\hline
\end{tabular}

\subsection{Application: recovery of the relative permittivity, reflection coefficient and transfer function.}

As already mentioned in the introductory section, the dispersive media in which the electromagnetic waves propagate, can be characterised by the relative permittivity:

$$
\epsilon_{r}(\omega)=\epsilon_{\infty}+\frac{\epsilon_{s}-\epsilon_{\infty}}{\left(1+\left(i \omega \tau_{0}\right)^{\alpha}\right)^{\beta}}
$$

in terms of the frequency variable $\omega$, for given $\epsilon_{\infty}, \epsilon_{s}, \tau_{0}, \alpha$ and $\beta$. It is of physical interest to study the associated reflection coefficient $(c f .[2])$ in magnitude:

$$
|\mathcal{R}(\omega)|=\left|\left(1-\sqrt{\epsilon_{r}(\omega)}\right) /\left(1+\sqrt{\epsilon_{r}(\omega)}\right)\right| .
$$

Another closely related notion is the transfer function $T(d, \omega)$ (see, e.g. [2,38-40]) given by

$$
T(d, \omega)=e^{\Upsilon(\omega) d}, \quad \Upsilon(\omega)=-i \omega \sqrt{\epsilon_{r}(\omega)} / c_{0}:=\Upsilon_{R}(\omega)+i \Upsilon_{I}(\omega),
$$

where $c_{0}=3.0 \times 10^{8}$ is the speed of light in free space. It describes the transfer rate of the electric field in frequency domain from the point $\boldsymbol{x}$ to the point $\boldsymbol{x}+d$ :

$$
\widehat{\boldsymbol{E}}(\boldsymbol{x}+d, \omega)=T(d, \omega) \widehat{\boldsymbol{E}}(\boldsymbol{x}, \omega),
$$

where $\widehat{\boldsymbol{E}}(\boldsymbol{x}, \omega)$ denotes the Fourier transform of the electric field $\boldsymbol{E}(\boldsymbol{x}, t)$.

In view of the above relations, one can compute $\boldsymbol{E}(\boldsymbol{x}, t)$ in time domain with fixed $\epsilon_{\infty}, \epsilon_{s}, \tau_{0}, \alpha$ and $\beta$ by solving the Maxwell's system (2.1)-(2.5), and then transform the field to the frequency domain. From (4.14), we can compute the approximate transfer function $\tilde{T}(d, \omega)$ in $\omega$ (as the field $\boldsymbol{E}$ is computed numerically), from which we can work out the approximate $\tilde{\epsilon}_{r}(\omega)$ and $|\tilde{\mathcal{R}}(\omega)|$ by using the relations (4.13) and (4.12), respectively. We are interested in fitting and recovering the analytic values of $\epsilon_{r}(\omega),|\mathcal{R}(\omega)|$ and $T(d, \omega)$ (evaluated exactly by (4.11) and (4.13) with given $\epsilon_{\infty}, \epsilon_{s}, \tau_{0}, \alpha$ and $\beta$ ) by the corresponding approximate values as in [2,38-40].

Similar to the setting in [2], we consider the Maxwell's system (2.1) in one spatial dimension with $z \in(a, b)$ and $t \in(0, T]$, but adding the source term $f(z, t):=E_{\text {inc }}(t) \chi_{z_{*}}(z)$ to the first equation of $(2.1)$. Here, $E_{\text {inc }}(t)$ is a modulated Gaussian pulse ( $c f .[2])$ :

$$
E_{\mathrm{inc}}(t)=e^{-a_{e}^{2}\left(t-4 / a_{e}\right)^{2}} \sin \left(2 \pi f_{e}\left(t-4 / a_{e}\right)\right) u(t),
$$

where $a_{e}=5 \times 10^{9} \mathrm{~s}^{-1}$, the central frequency $f_{e}=6 \mathrm{GHz}$, and $u(t)$ is the unit step function, i.e. $u(t)=1$ when $t \geq 0$ while $u(t)=0$ when $t<0$. Note that the energy of the pulse ranges from $0.1 \mathrm{GHz}$ to $10 \mathrm{GHz}$. In the source term, $z_{*} \in(a, b)$ is the location where the pulse is excited, and $\chi_{z_{*}}(z)=1$ at $z=z_{*}$, but it is equal to 0 elsewhere on $(a, b)$. It is noteworthy that the vector fields in the system (2.1) reduce to the scalar fields $E_{x}(z, t), H_{y}(z, t)$ and $P_{x}(z, t)$ in one dimension.

For clarity, we sketch the algorithm as follows. 

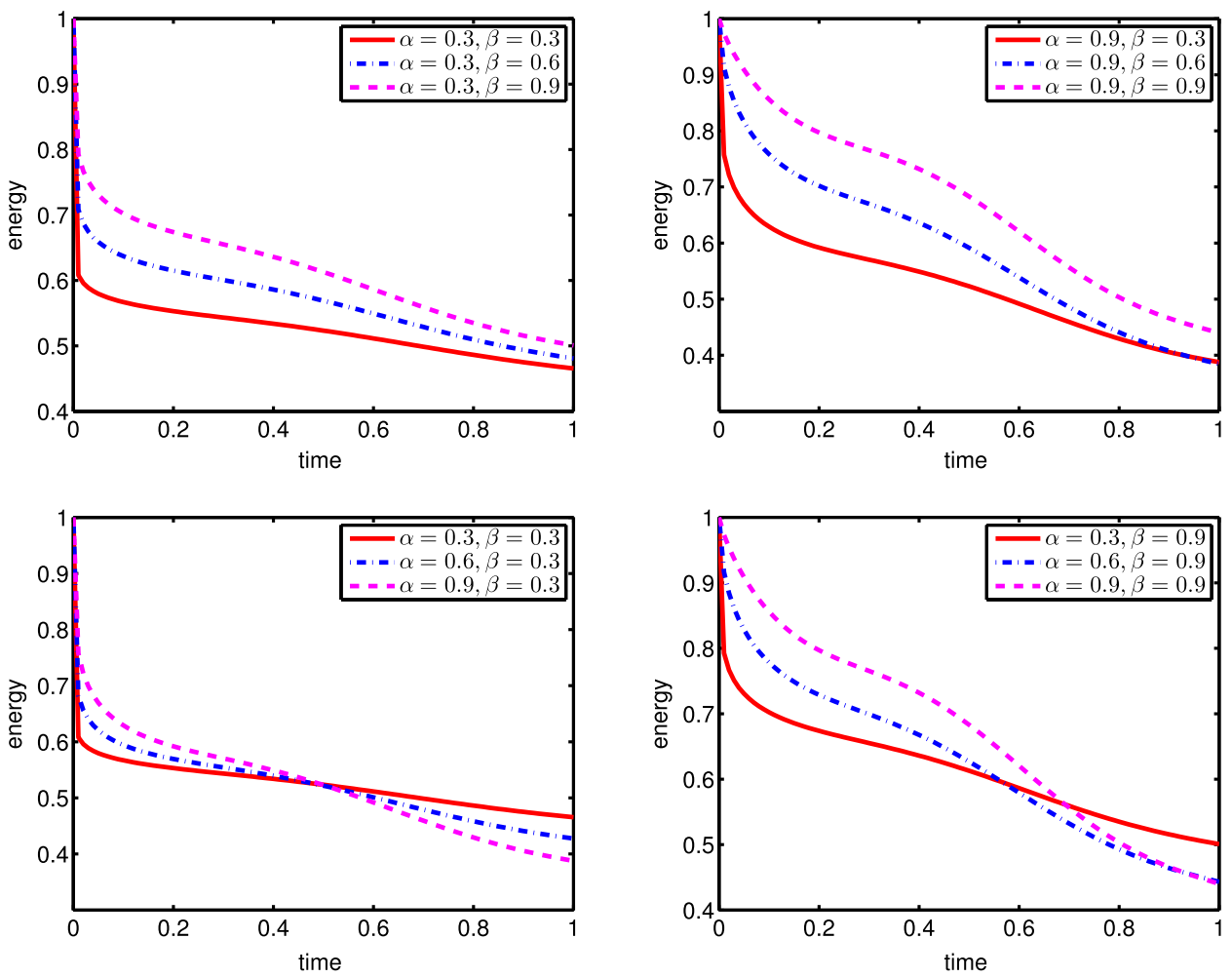

Figure 4. Evolution of the discrete energy $\mathscr{E}_{N}^{k}$ with different $\alpha$ and $\beta$.
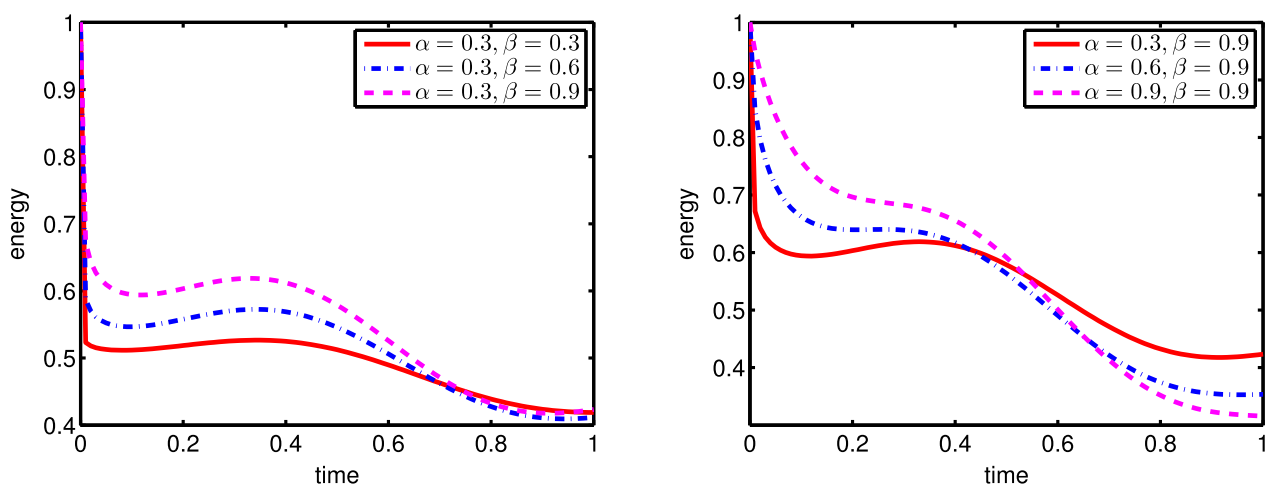

FiguRE 5. Evolution of the discrete energy $\tilde{\mathscr{E}}_{N}^{k}$ with different $\alpha$ and $\beta$.

(i) Solve the Maxwell's system for given $\epsilon_{\infty}, \epsilon_{s}, \tau_{0}, \alpha$ and $\beta$. Here, we adopt the finite-difference time-domain (FDTD) method to discretise the one dimensional system [2], but use the fractional integral formulation of the polarisation relation together with the aforementioned temporal discretisation, and fast convolution algorithm. With these, we can obtain the numerical approximation $E_{x, m}^{k}$ of $E_{x}(z, t)$ on the space-time grids: $t_{k}=k \Delta t$ and $z_{m}=a+m \Delta z$

(ii) Apply the discrete Fourier transform (cf. [12], p. 156) to $\left\{E_{x, m_{*}}^{k}\right\}_{k=1}^{N_{t}}$ and $\left\{E_{x, m_{*}+l}^{k}\right\}_{k=1}^{N_{t}}$ (at the locations $z_{*}=z_{m_{*}}$ and $z=z_{*}+d$ with $d=l \Delta z$ ) from the time domain to the frequency domain that leads to 


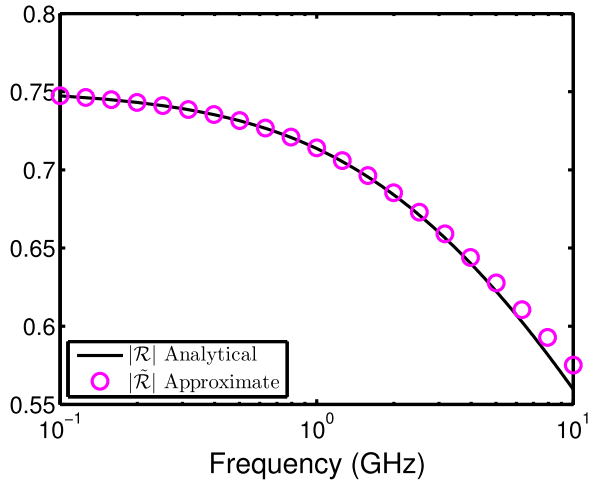

(a)

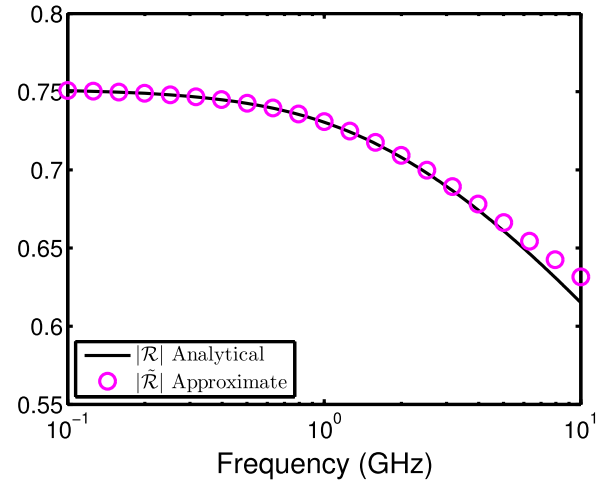

(b)

FIGURE 6. Real and imaginary part of the analytical magnitude of the reflection coefficient and the approximate value. (a) $\alpha=0.8, \beta=0.9$. (b) $\alpha=0.9, \beta=0.6$.

$\left\{\widehat{E}_{x, m_{*}}^{\omega_{j}}\right\}_{j=1}^{N_{\omega}}$ and $\left\{\widehat{E}_{x, m_{*}+l}^{\omega_{j}}\right\}_{j=1}^{N_{\omega}}$. Then the approximate transfer function is

$$
\tilde{T}\left(d, \omega_{j}\right)=\widehat{E}_{x, m_{*}+l}^{\omega_{j}} / \widehat{E}_{x, m_{*}}^{\omega_{j}} .
$$

(iii) Substitute (4.16) into (4.13) leading to the approximation:

$$
\tilde{\Upsilon}_{R}\left(\omega_{j}\right)=\ln \left(\left|\widehat{E}_{x, m_{*}+l}^{\omega_{j}} / \widehat{E}_{x, m_{*}}^{\omega_{j}}\right|\right) / d, \tilde{\Upsilon}_{I}\left(\omega_{j}\right)=\left(\arg \left\{\widehat{E}_{x, m_{*}+l}^{\omega_{j}}\right\}-\arg \left\{\widehat{E}_{x, m_{*}}^{\omega_{j}}\right\}\right) / d .
$$

Accordingly, we derive from (4.13) and (4.17) the real and the imaginary part of the approximate relative permittivity:

$$
\tilde{\epsilon}_{r}\left(\omega_{j}\right)=\tilde{\epsilon}^{\prime}\left(\omega_{j}\right)-i \tilde{\epsilon}^{\prime \prime}\left(\omega_{j}\right)=-\left(c_{0}\left(\tilde{\Upsilon}_{R}\left(\omega_{j}\right)+i \tilde{\Upsilon}_{I}\left(\omega_{j}\right)\right) / \omega_{j}\right)^{2},
$$

and from (4.12) the approximate magnitude of the reflection coefficient:

$$
\left|\tilde{\mathcal{R}}\left(\omega_{j}\right)\right|=\left|\left(1-\sqrt{\tilde{\epsilon}_{r}\left(\omega_{j}\right)}\right) /\left(1+\sqrt{\tilde{\epsilon}_{r}\left(\omega_{j}\right)}\right)\right| .
$$

In the computation, we take $a=0, b=1.1 \mathrm{~m}, T=5.304 \times 10^{-9} \mathrm{~s}, \epsilon_{s}=50, \epsilon_{\infty}=2, \tau_{0}=1.53 \times 10^{-10} \mathrm{~s}$, $\Delta t=1.768 \times 10^{-12} \mathrm{~s}, \Delta z=1.1 \mathrm{~mm}, z_{*}=0.55 \mathrm{~m}$, and sample $\left\{\omega_{j}\right\}_{j=1}^{N_{\omega}}$ in $(0.1,10) \mathrm{GHz}$ as in [2]. In Figure 6, we plot the analytical magnitude of the reflection coefficient $|\mathcal{R}(\omega)|$ and approximate values against samples of $\left\{\omega_{j}\right\}$ with different $\alpha, \beta$. In Figure 7 , we show the analytical complex transfer function $T\left(d, \omega_{j}\right)$ and its approximation $\tilde{T}\left(d, \omega_{j}\right)$ with different $\alpha, \beta$ and $d$. We observe a better approximation than that in [2], which shows the advantage of our approach. In Figure 8, we depict the complex relative permittivity $\epsilon_{r}=\epsilon^{\prime}-i \epsilon^{\prime \prime}$ and the approximate $\tilde{\epsilon}_{r}=\tilde{\epsilon}^{\prime}-i \tilde{\epsilon}^{\prime \prime}$ with different $\alpha$ and $\beta$, which is not presented in [2]. Indeed, we observe a good agreement of the exact and numerical values.

\section{CONCLUding REMARKS}

In this paper, we rigorously showed the energy dissipation law and $L^{2}$-stability of the Maxwells equations in a $\mathrm{H}-\mathrm{N}$ dispersive medium, which were unavailable in any literature. We proposed a backward Euler-type time discretisation based on the piecewise constant approximation of the induced fractional electric polarisation relation. With a delicate study of the discrete weights resulted from the approximation of this relation, we 


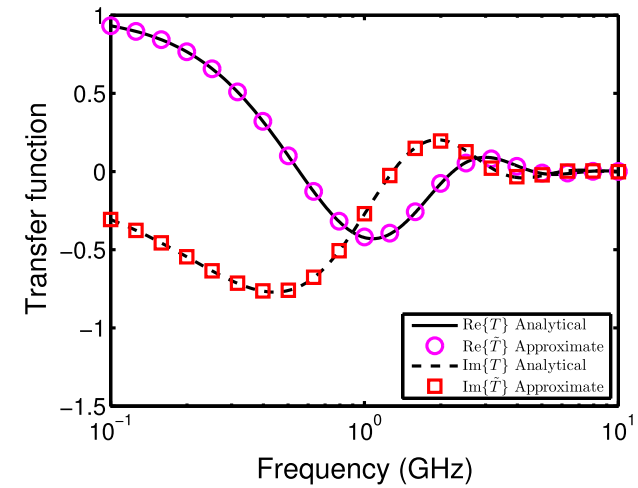

(a)

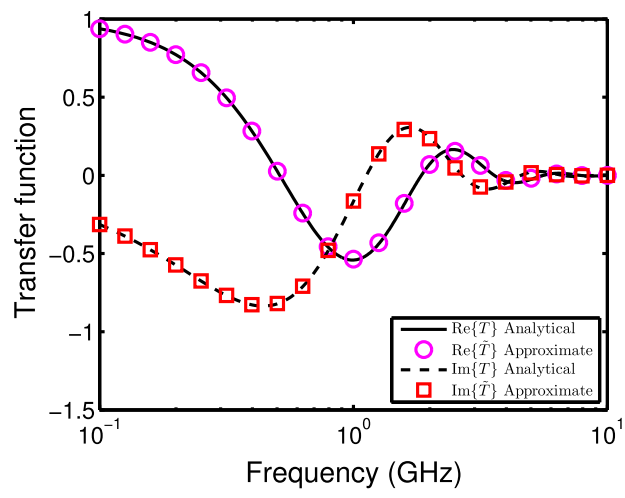

(c)

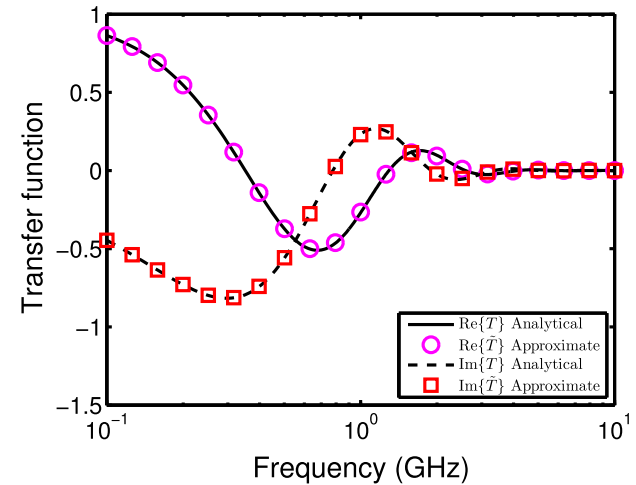

(b)

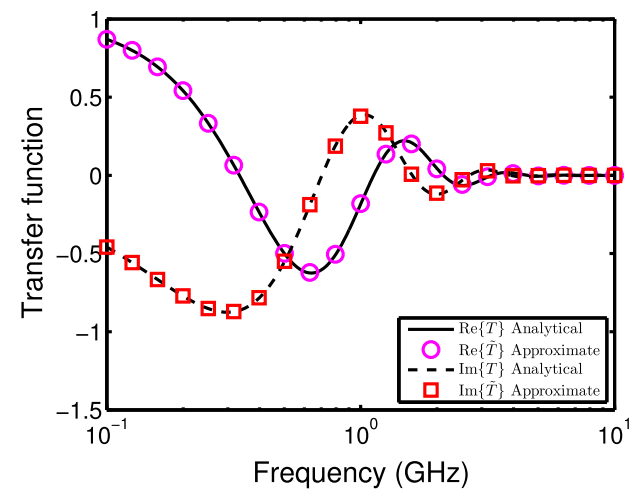

(d)

FiguRE 7. Real and imaginary part of the analytical complex transfer function and the approximate one of the H-N medium. Left: $d=20 \Delta z$; Right: $d=30 \Delta z$. (a) $\alpha=0.8, \beta=0.9$. (b) $\alpha=0.8, \beta=0.9$. (c) $\alpha=0.9, \beta=0.6$. (d) $\alpha=0.9, \beta=0.6$.

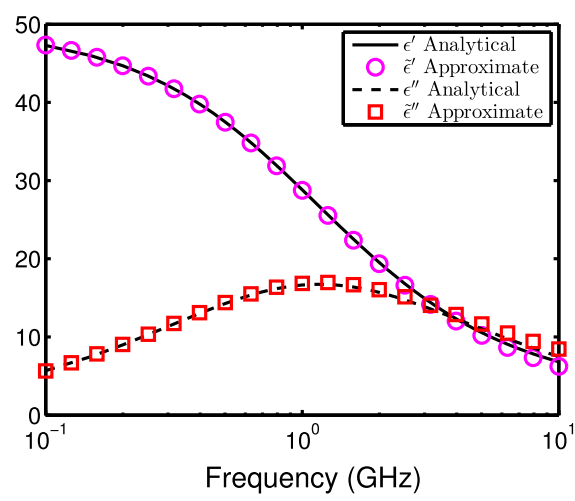

(a)

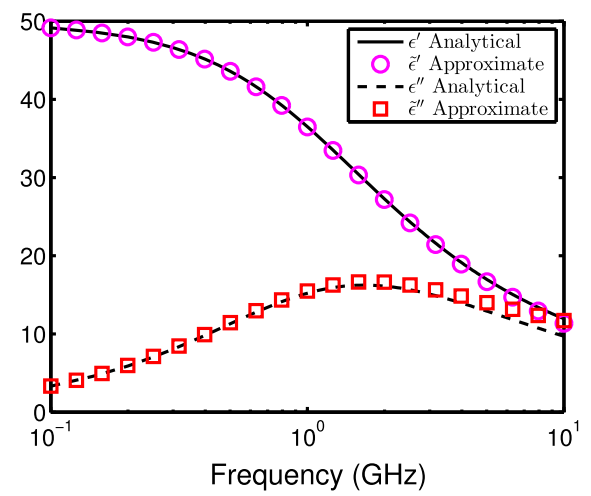

(b)

FiguRE 8. Real and imaginary part of the analytical complex relative permittivity and the approximate one of the H-N media. (a) $\alpha=0.8, \beta=0.9$. (b) $\alpha=0.9, \beta=0.6$. 
proved the semi-discrete scheme satisfies an analogous (modified) energy dissipation law, so we further showed the unconditional stability and convergence of the semi-discretised scheme. We then introduced a fast convolution algorithm so that the time-stepping algorithm can incorporate with various spatial discretisation such as finite elements, finite differences and spectral elements. As an illustration, we considered the full-discretisation scheme for the two-dimensional model with the spatial discretisation by a spectral-Galerkin method, and conducted the error analysis. We provided ample numerical results to show the efficiency and robustness of the proposed method. We also applied the solver to a real application in the recovery of the relative permittivity, reflection coefficient and transfer function.

There are some related issues worthy of future investigation. Here, we developed the first-order time discretisation scheme, but the generalisation to higher order schemes appears far from trivial. One challenge lies in how to show the important property of the weights similar to that in Lemma 3.1. In this work, we only implemented and analysed the spatial discretisation for two-dimensional problems, but it is of more practical interest to consider the full three-dimensional model using e.g. the edge elements.

\section{Appendix A. Properties of the Mittag-Leffler function}

We collect below some properties of the ML function that are used in this paper.

- According to [18], (5.1.6), the Laplace transform of the ML function in (2.8) takes the form

$$
\mathscr{L}\left[t^{\mu-1} E_{\rho, \mu}^{\gamma}\left(\sigma t^{\rho}\right)\right](s)=\frac{s^{-\mu}}{\left(1-\sigma s^{-\rho}\right)^{\gamma}}=\frac{s^{\rho \gamma-\mu}}{\left(s^{\rho}-\sigma\right)^{\gamma}}, \quad \text { for } \rho, \mu>0 \text { and real } \gamma
$$

- We have the integral identity (cf. [15], (4), (5) or [18], (5.1.15), (5.1.19)):

$$
\int_{0}^{z} t^{\mu-1} E_{\rho, \mu}^{\gamma}\left(\sigma t^{\rho}\right) \mathrm{d} t=z^{\mu} E_{\rho, \mu+1}^{\gamma}\left(\sigma z^{\rho}\right), \quad \text { for } \rho, \mu>0 \text { and real } \gamma .
$$

- We know from [35], page 9 that for all $\rho>0$ and real $\gamma, \mu$, the Mittag-Leffler function with three parameters $E_{\rho, \mu}^{\gamma}(z)$ is bounded in a finite interval, i.e.

$$
\left|E_{\rho, \mu}^{\gamma}(z)\right| \leq M, \quad \forall z \in[a, b],
$$

where $a, b$ and $M>0$ are constants.

- For all $\sigma>0, t^{\mu-1} E_{\rho, \mu}^{\gamma}\left(-\sigma t^{\rho}\right)$ is completely monotonic, if only if $0<\rho<\mu \leq 1$ and $0<\gamma \leq \mu / \rho(c f$. [18], (5.1.10)), that is,

$$
(-1)^{n} \frac{\mathrm{d}^{n}}{\mathrm{~d} t^{n}}\left[t^{\mu-1} E_{\rho, \mu}^{\gamma}\left(-\sigma t^{\rho}\right)\right] \geq 0, \quad \forall t \in(0, \infty),
$$

where we refer to Definition 3.22 of [18] for the definition of the completely monotonicity function.

Acknowledgements. The research of Yubo Yang is partially supported by the key research projects of Nanhu College, Jiaxing University under grant N41472001-40. The research of Li-Lian Wang is partially supported by Singapore MOE AcRF Tier 2 Grants: MOE2017-T2-144 \& MOE2018-T2-1-059. The research of Fanhai Zeng is partially supported by the startup fund from Shandong University (No. 11140082063130). Yubo Yang would like to thank Nanyang Technological University for hosting his visit devoted to this collaborative work.

\section{REFERENCES}

[1] A. Alegria, L. Goitiandia, I. Telleria and J. Colmenero, $\alpha$-relaxation in the glass-transition range of amorphous polymers. 2. Influence of physical aging on the dielectric relaxation. Macromolecules 30 (1997) 3881-3888.

[2] C.S. Antonopoulos, N.V. Kantartzis and I.T. Rekanos, FDTD method for wave propagation in Havriliak-Negami media based on fractional derivative approximation. IEEE T. Magn. 53 (2017) 1-4. 
[3] P. Bia, D. Caratelli, L. Mescia, R. Cicchetti, G. Maione and F. Prudenzano, FDTD method for wave propagation in HavriliakNegami media based on fractional derivative approximation. Sign. Process. 107 (2015) 312-318.

[4] K. Biswas, G. Bohannan, R. Caponetto, A.M. Lopes and J.A.T. Machado, Fractional-order models of vegetable tissues. In: Fractional-Order Devices. Springer (2017) 73-92.

[5] M.F. Causley, P.G. Petropoulos and S. Jiang, Incorporating the Havriliak-Negami dielectric model in the FDTD method. J. Comput. Phys. 230 (2011) 3884-3899.

[6] J. Chakarothai, Novel FDTD scheme for analysis of frequency-dependent medium using fast inverse Laplace transform and Prony's method. IEEE Trans. Antennas Propag. 67 (2019) 6076-6089.

[7] G. Cohen and S. Pernet, Finite Elements and Discontinuous Galerkin Methods for Transient Wave Equations. Springer Series in Scientific Computation. Springer (2017).

[8] K.S. Cole and R.H. Cole, Dispersion and absorption in dielectrics I. Alternating current characteristics. J. Chem. Phys. 9 (1941) 341-351.

[9] D.W. Davidson and R.H. Cole, Dielectric relaxation in glycerol, propylene glycol, and n-propanol. J. Chem. Phys. 19 (1951) 1484-1490.

[10] P.J.W. Debye, Polar Molecules. Dover (1929).

[11] L. Demkowicz, Computing with hp-Adaptive Finite Elements: Vol. 1. One- and Two-Dimensional Elliptic and Maxwell Problems. Chapman and Hall/CRC (2006).

[12] A.Z. Elsherbeni and V. Demir, The Finite-Difference Time-Domain Method for Electromagnetics with MATLAB Simulations. SciTech, Edison, NJ, USA (2015).

[13] A. Garcia-Bernabé, R.D. Calleja, M. Sanchis, A. Del Campo, A. Bello and E. Pérez, Amorphous-smectic glassy main chain LCPs. II. Dielectric study of the glass transition. Polymer 45 (2004) 1533-1543.

[14] R. Garrappa, Numerical evaluation of two and three parameter Mittag-Leffler functions. SIAM J. Numer. Anal. 53 (2015) $1350-1369$.

[15] R. Garrappa and G. Maione, Fractional Prabhakar derivative and applications in anomalous dielectrics: a numerical approach. Lecture Notes Electr. Eng. 407 (2017) 429-439.

[16] R. Garrappa, F. Mainardi and M. Guido, Models of dielectric relaxation based on completely monotone functions. Fract. Calc. Appl. Anal. 19 (2016) 1105-1160.

[17] A. Giusti, I. Colombaro, R. Garra, R. Garrappa, F. Polito, M. Popolizio and F. Mainardi, A practical guide to Prabhakar fractional calculus. Fract. Calc. Appl. Anal. 23 (2020) 9-54.

[18] R. Gorenflo, A.A. Kilbas, F. Mainardi and S.V. Rogosin, Mittag-Leffler Functions, Related Topics and Applications. Springer, Berlin (2014).

[19] S. Havriliak and S. Negami, A complex plane analysis of $\alpha$-dispersions in some polymer systems. J. Polym. Sci. C 14 (1966) 99-117.

[20] S. Havriliak and S. Negami, A complex plane representation of dielectric and mechanical relaxation processes in some polymers. Polymer 8 (1967) 161-210.

[21] C. Huang and L.-L. Wang, An accurate spectral method for the transverse magnetic mode of Maxwell equations in Cole-Cole dispersive media. Adv. Comput. Math. 45 (2019) 707-734.

[22] D.F. Kelley, Piecewise linear recursive convolution for the FDTD analysis of propagation through linear isotropic dispersive dielectrics. Ph.D. thesis, Pennsylvania State University (1999).

[23] D.F. Kelley, T.J. Destan and R.J. Luebbers, Debye function expansions of complex permittivity using a hybrid particle swarmleast squares optimization approach. IEEE T. Antenn. Propag. 55 (2007) 1999-2005.

[24] A.A. Kilbas, M. Saigo and R.K. Saxena, Generalized Mittag-Leffler function and generalized fractional calculus operators. Integr. Transf. Spec. F. 15 (2004) 31-49.

[25] J. Li and Y. Huang, Time-Domain Finite Element Methods for Maxwell's Equations in Metamaterials. Springer Series in Computational Mathematics. Springer (2013).

[26] J. Li, Y. Huang and Y. Lin, Developing finite element methods for Maxwell's equations in a Cole-Cole dispersive medium. SIAM J. Sci. Comput. 33 (2011) 3153-3174.

[27] A.M. Lopes, J.T. Machado and E. Ramalho, Fractional-order model of wine. In: Chaotic, Fractional, and Complex Dynamics: New Insights and Perspectives. Springer (2018) 191-203.

[28] C. Lubich and A. Schädle, Fast convolution for nonreflecting boundary conditions. SIAM J. Sci. Comput. 24 (2002) 161-182.

[29] W. McLean, V. Thomée and L.B. Wahlbin, Discretization with variable time steps of an evolution equation with a positive-type memory term. J. Comput. Appl. Math. 69 (1996) 49-69.

[30] L. Mescia, P. Bia and D. Caratelli, Fractional derivative based FDTD modeling of transient wave propagation in HavriliakNegami media. IEEE Trans. Microwave Theory Tech. 62 (2014) 1920-1929.

[31] P. Monk, Finite Element Methods for Maxwell's Equations. Oxford University Press (2003).

[32] P. Monk, A comparison of three mixed methods for the time-dependent Maxwell's equations. SIAM J. Sci. Stat. Comput. 13 (1992) 1097-1122.

[33] I. Podlubny, Fractional Differential Equations: An Introduction to Fractional Derivatives, Fractional Differential Equations to Methods of their Solution and some of their Applications. Academic, San Diego, CA (1999).

[34] C. Polk and E. Postow, Handbook of Biological Effects of Electromagnetic Fields. CRC Press (1995).

[35] T.R. Prabhakar, A singular integral equation with a generalized Mittag-Leffler function in the kernel. J. Yokohama Math. 19 (1971) 7-15. 
[36] A. Quarteroni and A. Valli, Numerical Approximation of Partial Differential Equations. Springer, Berlin (1994).

[37] G.G. Raju, Dielectrics in Electric Fields. CRC Press, New York (2016).

[38] I.T. Rekanos, An auxiliary differential equation method for FDTD modeling of wave propagation in Cole-Cole dispersive media. IEEE Trans. Antennas Propag. 58 (2012) 3666-3674.

[39] I.T. Rekanos, FDTD modeling of Havriliak-Negami media. IEEE Microw. Wirel. Co. 22 (2012) 49-51.

[40] I.T. Rekanos, FDTD schemes for wave propagation in Davidson-Cole dispersive media using auxiliary differential equations. IEEE Trans. Antennas Propag. 60 (2012) 1467-1478.

[41] T. Repo and S. Pulli, Application of impedance spectroscopy for selecting frost hardy varieties of English ryegrass. Ann. Botany 78 (1996) 605-609.

[42] A. Schonhals, Fast calculation of the time dependent dielectric permittivity for the Havriliak-Negami function. Acta Polym. 42 (1991) 149-151.

[43] J.W. Schuster and R.J. Luebbers, An FDTD algorithm for transient propagation in biological tissue with a Cole-Cole dispersion relation. Proc. IEEE Antennas Propag. Soc. Int. Symp. 4 (1998) 1988-1991.

[44] J. Shen, T. Tang and L.-L. Wang, Spectral Methods: Algorithms, Analysis and Applications. Springer (2011).

[45] A. Taflove and S.C. Hagness, Computational Electrodynamics: The Finite-Difference Time-Domain Method. Artech House, London (2005).

[46] F. Torres, P. Vaudon and B. Jecko, Application of new fractional derivatives to the FDTD modeling of pulse propagation in a Cole-Cole medium. Microwave Opt. Technol. 13 (1996) 300-304.

[47] K. Xu and S. Jiang, A bootstrap method for sum-of-poles approximations. J. Sci. Comput. 55 (2013) $16-39$.

[48] F. Zeng, I. Turner and K. Burrage, A stable fast time-stepping method for fractional integral and derivative operators. J. Sci. Comput. 77 (2018) 283-307. 